\title{
Coordinated Hard Sphere Mixture (CHaSM): A simplified model for oxide and silicate melts at mantle pressures and temperatures
}

\author{
Aaron S. Wolf ${ }^{\mathrm{a}, \mathrm{b}, *}$, Paul D. Asimow ${ }^{\mathrm{b}}$, David J. Stevenson ${ }^{\mathrm{b}}$ \\ ${ }^{a}$ University of Michigan, Ann Arbor, Earth and Environmental Sciences Department, MI 48104 \\ ${ }^{b}$ California Institute of Technology, Division of Geological and Planetary Sciences, Pasadena CA 91125
}

\begin{abstract}
We develop a new model to understand and predict the behavior of oxide and silicate melts at extreme temperatures and pressures, including deep mantle conditions like those in the early Earth magma ocean. The Coordinated Hard Sphere Mixture (CHaSM) is based on an extension of the hard sphere mixture model, accounting for the range of coordination states available to each cation in the liquid. By utilizing approximate analytic expressions for the hard sphere model, this method is capable of predicting complex liquid structure and thermodynamics while remaining computationally efficient, requiring only minutes of calculation time on standard desktop computers. This modeling framework is applied to the $\mathrm{MgO}$ system, where model parameters are trained on a collection of crystal polymorphs, producing realistic predictions of coordination evolution and the equation of state of $\mathrm{MgO}$ melt over a wide range of pressures and temperatures. We find that the typical coordination number of the $\mathrm{Mg}$ cation evolves continuously upward from 5.25 at $0 \mathrm{GPa}$ to 8.5 at $250 \mathrm{GPa}$. The results produced by CHaSM are evaluated by comparison with predictions from published first-principles molecular dynamics calculations, indicating that CHaSM is accurately capturing the dominant physics controlling the behavior of oxide melts at high pressure. Finally, we present a simple quantitative model to explain the universality of the increasing grüneisen parameter trend for liquids, which directly reflects their pro-
\end{abstract}

\footnotetext{
*Corresponding author: aswolf@umich.edu

Email addresses: aswolf@umich.edu (Aaron S. Wolf), asimow@gps.caltech.edu (Paul D. Asimow), djs@gps . caltech.edu (David J. Stevenson)
}

Preprint submitted to Geochimica et Cosmochimica Acta

April 4, 2015 
gressive evolution toward more compact solid-like structures upon compression. This general behavior is opposite that of solid materials, and produces steep adiabatic thermal profiles for silicate melts, thus playing a crucial role in magma ocean evolution.

Keywords: High Pressure Melts, Silicate Liquids, Grüneisen Parameter, Magma

Ocean, Liquid Thermodynamics

\section{Introduction}

Understanding the behavior of silicate and oxide liquids at mantle pressures is crucial to reconstructing evolution of Earth and other terrestrial planets, both today and in the distant past. While molten silicates are primarily restricted to the shallow mantle today, their material properties directly determined the Earth's evolutionary path as it cooled from a nearly whole-mantle magma ocean to its present-day solid state.

Recent first-principles theoretical calculations and experimental shock-wave investigations (Stixrude et al., 2009; Mosenfelder et al., 2009) indicate that the adiabatic profiles of molten $\mathrm{MgSiO}_{3}$ as well as a wide collection of other silicate melts (Thomas et al., 2012; Asimow, 2012)) are significantly steeper than previously thought. Giant impact simulations indicate that events like the moon-forming impact efficiently deliver heat to great depths, supporting the possibility of a deep potentially whole-mantle magma ocean early in Earth's history (Nakajima and Stevenson, 2014). The evolution of this planetary-scale magma ocean as it cools and crystallizes is dominated by the poorly understood behavior of silicates at extreme pressures and temperatures. Probing these conditions is difficult for both theory and experiment, and further challenges are posed by the large relevant compositional space, including at least $\mathrm{MgO}, \mathrm{SiO}_{2}$, and spin-dependent FeO. The primary tool for computational work, first-principles molecular dynamics (FPMD), has been used to probe the behavior of a variety of silicate liquid compositions across the $\mathrm{MgO}-\mathrm{SiO}_{2}$ binary (e.g. Stixrude and Karki, 2005; Karki et al., 2006, 2007; de Koker et al., 2008, 2013) as well as iron-rich silicate liquid $\mathrm{Fe}_{2} \mathrm{SiO}_{4}$ (Muñoz Ramo and Stixrude, 2014). While extremely powerful, this approach has limitations including high computational cost, lower bounds on temperature due to relaxation constraints, as well as restrictions to simulation length and time scales that 
are many orders of magnitude smaller than those relevant to the Earth or experimental methods. These limitations invite the parallel development of new theoretical methods for investigating the behavior of molten oxides and silicates.

Though there is much about the structure of high-pressure silicate melts that we do not yet understand, there is a growing consensus amongst experimental and theoretical studies that the evolution of cation coordination number plays a critical role in silicate liquid compression. Nuclear Magnetic Resonance (NMR) spectroscopy probes the local bonding environment around particular target atoms within a material. Numerous ${ }^{29} \mathrm{Si}-\mathrm{NMR}$ and ${ }^{27} \mathrm{Al}-\mathrm{NMR}$ studies over the last two decades have examined the evolution of cation coordination number (signifying the number of oxygens bonded to each cation), showing its smooth increase with compression from 4 to 6 for these network forming cations based on measurements of quenched glasses, thought to represent frozen snapshots of the liquid structure (e.g., Xue et al., 1991; Yarger et al., 1995; Lee, 2011). These ex-situ spectroscopy studies on glasses are nicely complimented by in-situ melt studies that measure diffuse $\mathrm{x}$-ray diffraction signals for laseror furnace-heated samples at high pressures. With carefully designed experiments for the cubic-anvil press (Funamori et al., 2004), Paris-Edinburgh press (Yamada et al., 2011; Wang et al., 2014), and diamond anvil cell (Sanloup et al., 2013), recent studies have measured in-situ x-ray structure factors for silicate melts over a range of elevated pressures. By transforming and integrating these data, they found upward evolution of cation coordination with compression. These experimental findings all compare well with FPMD calculations, which show progressive cation coordination evolution for all cations within the melt, indicating that coordination evolution must play a major role in accommodating compression for oxide and silicate melts over a wide range of conditions.

As a complement to accurate first-principles calculations, we have developed the Coordinated Hard Sphere Mixture (CHaSM), which represents oxide and silicate liquids by tracking the relative populations of differently coordinated cation species (clusters) as a function of temperature and pressure. This model is based on extending the standard hard sphere mixture model (Mansoori et al., 1971), which was recently applied to silicate liquids by Jing and Karato (2011). In order to reasonably represent 
compression-induced changes to internal energy, we implement a perturbation to the standard hard sphere model, in which the internal energy is represented by empirical cluster potentials that are coordination dependent. Using entirely analytic expressions and the principle of free energy minimization, we determine the relative populations of the different coordination states. We also demonstrate how the equations of state for a set of solid structures can be used to train appropriate values for the cluster potentials, thus providing a fully predictive model for oxide liquids that does not require fitting any liquid data.

Shock wave experiments indicate that all silicate liquids maintain much steeper adiabatic profiles at extreme pressures than their solid counterparts (Thomas et al., 2012; Asimow, 2012). This is a consequence of the compression behavior of the thermodynamic grüneisen parameter $\gamma$ :

$$
\left.\frac{d T}{d P}\right|_{S}=\gamma\left(\frac{T}{K_{S}}\right)
$$

where $\gamma$ gives the proportionality constant for the adiabatic temperature path and $K_{S}$ is the adiabatic bulk modulus. The function $\gamma(V)$ is thus a crucial property for understanding early Earth evolution, as it largely determines the thermal profile through the convecting (nearly adiabatic) magma ocean. Both theoretical calculations and experiments show that the grüneisen parameter increases upon compression not just for silicate melts but for nearly all liquids (McQueen and Marsh, 1960; Knopoff and Shapiro, 1970; Boehler and Kennedy, 1977), which is opposite to the well-understood behavior of solid materials. Unfortunately, we do not yet have a mechanistic understanding for why this property behaves so counterintuitively. We propose a simple and entirely general explanation, which provides accurate quantitative predictions along with important qualitative insights about the general properties of liquids.

We begin this paper by first reviewing the physics behind the Hard Sphere model, previous geological applications, and the variational perturbation approach in Section 2. In Section 3, we present the framework for our new model, the Coordinated Hard Sphere Mixture (CHaSM), taking special care to explicitly connect the model parameters to the physical aspects of oxide melt structure. In Section (4), we present a first application of $\mathrm{CHaSM}$ to the $\mathrm{MgO}$ melt system, demonstrating how its parameters can 
be trained on solid data in order to obtain predictions of melt behavior over a wide pressure-temperature range. In Section 5, we review the existing experimental and computational evidence considering the grüneisen parameter behavior of liquids and present a simple model that provides a general explanation of these data, while also providing testable quantitative predictions. Finally in Section 6, we review our findings and discuss future prospects for this new modeling effort.

\section{The Hard Sphere Model}

Unlike crystalline solids, whose compression behavior is dominated by the energetic cost of bond-length shortening, liquid equations of state are influenced by their compression-induced structural evolution. The Hard Sphere model successfully describes simple liquids based on its approximate representation of atomic spatial distributions, capturing the associated configurational entropies and how they evolve with pressure.

\subsection{Intro to Hard Spheres and Simple Liquids}

In a hard sphere fluid, liquid particles are described as a collection of non-overlapping and non-deformable spheres, reflecting the short-range electronic repulsive forces that give atoms their finite size. Like the ideal gas this model is devoid of energy interactions, and thus obtains its rich behavior entirely from entropic and kinetic energy terms. The details of the basic hard sphere model are readily found in statistical physics textbooks, but we reproduce all the necessary equations in Appendix A.

Absence of long-range order is a defining characteristic of liquids, and thus their structure must be characterized probabilistically. The Radial Distribution Function (RDF), denoted $g(r)$, is used to describe the time-averaged liquid structure, expressing how particle density varies with separation distance:

$$
g(r) \equiv \frac{\rho(r)}{\langle\rho\rangle} \quad \text { and } \quad \mathcal{P}(r) d r=4 \pi r^{2}\langle\rho\rangle g(r) d r
$$

The RDF is computed in atomic simulations by averaging the number of particles lying within spherical shells over a range of sizes. 

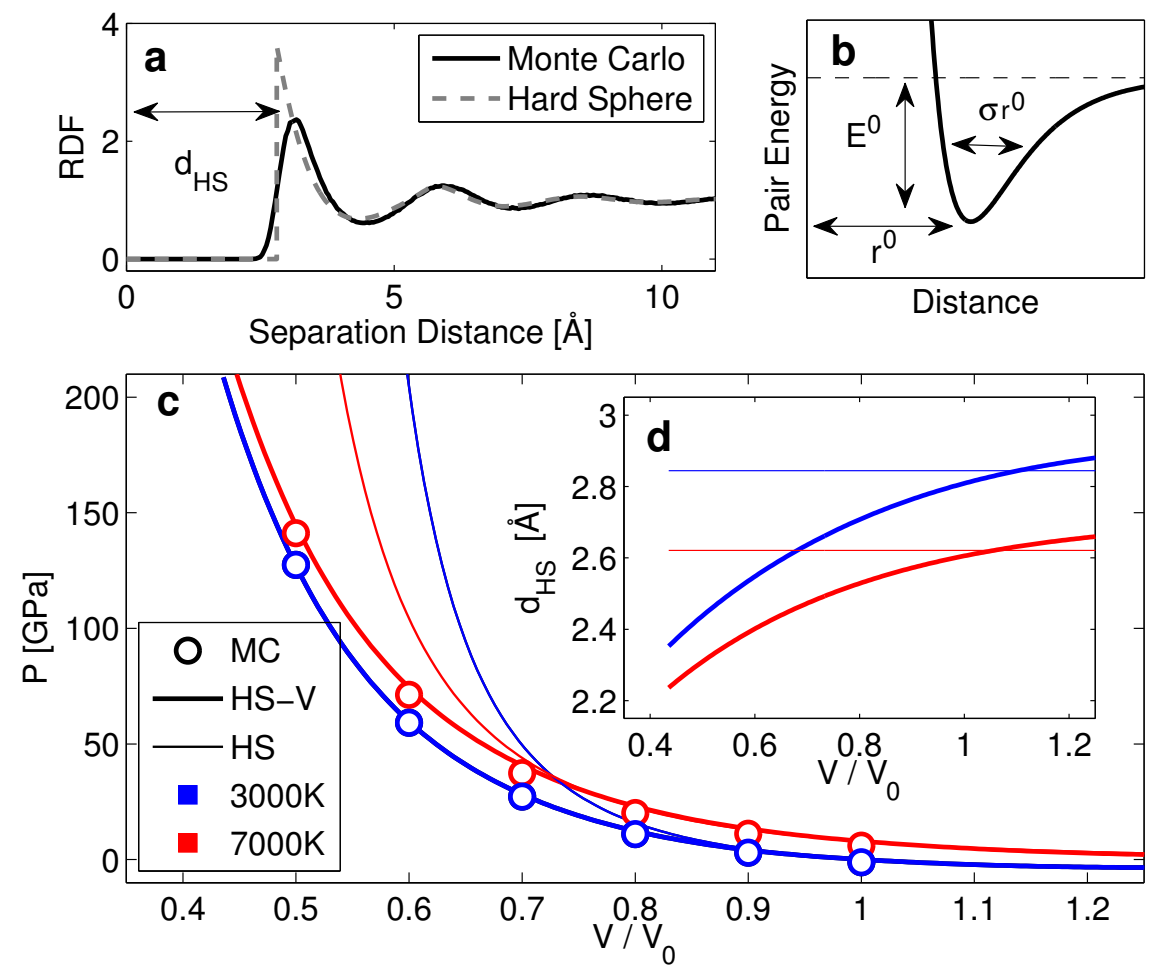

Figure 1: Comparison of the standard Hard Sphere (HS) and variational Hard Sphere (HS-V) models to Monte Carlo (MC) simulations for a toy single-component melt tuned to have compression behavior similar to $\mathrm{MgO}$ at $3000 \mathrm{~K}$. Panel (a) compares the Radial Distribution Function (RDF) curves from the HS and MC models; (b) illustrates the pair potential parameters, defining the energy well position $r^{0}$, depth $E^{0}$, and width $\sigma r^{0}$; (c) compares the equation of state predictions for the MC, HS, and HS-V models at $3000 \mathrm{~K}$ and $7000 \mathrm{~K}$; and (d) shows the evolution of $d_{\mathrm{HS}}$ for the HS and HS-V models in light and heavy weight lines, respectively.

To demonstrate the hard sphere model, we compare its predictions to those of atomic simulation, where atomic positions and their effect on total energy are directly modeled. Unlike the hard sphere model, atomic simulations account for interaction energies by representing them with either first-principles quantum mechanics or empirical potentials, which describe the shape of the energy well for pairwise atomic interactions (see Figure 1b). These simulations can either use the molecular dynamics (MD) or molecular Monte Carlo (MC) approaches, where atomic positions are varied according to either Newtonian mechanics or random proposals, respectively. Both methods pro- 
duce consistent predictions of liquid structure and thermodynamics, though dynamical quantities like diffusivity or viscosity are typically restricted to MD simulations.

For a simple demonstration, we explore a single-component reference liquid whose pair potential has been chosen to reproduce the $3000 \mathrm{~K}$ isotherm of $\mathrm{MgO}$ melt (Karki et al., 2006). This toy example shows the general characteristics of liquids while retaining temperature and pressure scales appropriate to silicate and oxide melts. Figure 1(a) compares the RDF curve from the Hard Sphere model to the precise structure obtained from empirical Monte Carlo simulation (using a basic random-walk Monte Carlo scheme in MATLAB). Both curves demonstrate the primary structural features of all liquids: a sharp inner edge to the particle density and decaying density oscillations with increasing distance. The inner edge is a direct consequence of finite particle size resulting from repulsive interactions, and is thus well represented by the hard sphere diameter $d_{H S}$. The density oscillations reflect the liquid's short-range order, where each subsequent maximum corresponds to a shell of next-nearest neighboring particles. At great distance, all ordering is lost as the density asymptotically approaches the average bulk value, with $g(r)=1$. The hard sphere model achieves this successful approximation of liquid structure with only a single free parameter, $d_{H S}$. Furthermore, its an entirely analytic model (see Appendix A), resulting in nearly instantaneous calculations in contrast to the long convergence times needed for atomic simulations.

In addition to structure, the hard sphere model also estimates thermodynamic properties like pressure and Helmholtz Free energy by estimating the entropy associated with the predicted liquid structure. The isothermal equation of state is shown in Figure 1c for both the HS model and MC simulations, in the light weight curves and open symbols, respectively. The $3000 \mathrm{~K}$ and $7000 \mathrm{~K}$ hard sphere diameters, shown by the light weight lines in panel $\mathrm{d}$, were determined by matching the reference bulk modulus $^{1}$. The HS model matches the MC results well in the low pressure-region, but begins to deviate significantly at $3000 \mathrm{~K}$ and $7000 \mathrm{~K}$ for pressures exceeding $10 \mathrm{GPa}$

\footnotetext{
${ }^{1}$ The basic HS model curves in Figure 1 include an additional background potential term to roughly capture the attractive Coulombic field. This negative pressure term is important for representing near-ambient pressure properties.
} 
and $40 \mathrm{GPa}$, respectively. The failure of the basic hard sphere model to extend to high pressures reflects the inadequacy of assuming fixed hard sphere sizes.

\subsection{Variational Approach to Hard Spheres}

The variational hard sphere model, developed by Mansoori and Canfield (1969), address this limitation by adding a structural energy perturbation to the hard sphere model, which is used to estimate the structure and entropy of the liquid. The hard sphere size is then determined by minimizing the total Helmholtz free energy (with respect to its value):

$$
F^{\mathrm{HS}-\mathrm{V}}(V, T)=\min _{d_{H S}}\left\{F^{\mathrm{HS}}\left(V, T, d_{H S}\right)+E^{\mathrm{struc}}\left(V, d_{H S}\right)\right\}
$$

This approach has the advantage of eliminating hard sphere size as a free parameter, allowing the model to determine its optimal value.

To estimate the structural energy, we must choose a pair potential to describe the pairwise interactions. Though many potential functional forms exist (e.g. LennardJones, Buckingham, Mie, etc.), we use the Morse potential for its flexibility and easily interpreted tunable parameters:

$$
\begin{array}{r}
E_{*}^{\mathrm{Morse}}\left(r_{*}\right)=\exp \left[-2 \frac{\left(r_{*}-1\right)}{\sigma}\right]-2 \exp \left[-\frac{\left(r_{*}-1\right)}{\sigma}\right] \\
\text { with, } E_{*}=E / E^{0} \text { and } r_{*}=r / r^{0}
\end{array}
$$

where the energy and length scales defining the depth and position of the energy well are given by $E^{0}$ and $r^{0}$, and the normalized width of the energy well is defined by $\sigma$ (see Figure 1b). The total structural energy is then just an RDF-weighted average over pairwise particle interactions:

$$
\begin{aligned}
E^{\text {struc }} & =\frac{1}{2} \int_{0}^{\infty} E^{\mathrm{pot}}(r) \mathcal{P}(r) d r \\
& =2 \pi\langle\rho\rangle \int_{0}^{\infty} E^{\mathrm{pot}}(r) r^{2} g(r) d r
\end{aligned}
$$

where $E^{\text {pot }}$ is the pair potential and the factor of $\frac{1}{2}$ prevents double counting.

Standard numerical minimization of the total free energy (Equation 3) is used to infer the appropriate hard sphere sizes, shown by the heavy-weight lines in Figure 1d, 
which are seen to shrink with both increasing temperature and pressure. This is intuitively reasonable since reducing the hard sphere size is accomplished by climbing up the short-range repulsive slope of the potential, which requires energy that can be provided either thermally or by doing mechanical work on the system. The free energy surface as a function of volume and temperature is easily calculated, and numerical derivatives yield desired thermodynamic quantities, such as entropy, pressure, compressibility, etc. In Figure 1(c), we can see that the variational method extends the hard sphere model's accuracy across the entire explored pressure range. By eliminating all free parameters, the variational approach thus transforms the hard sphere model from an empirical data-fitting procedure into a predictive model for simple liquids.

\subsection{Previous Application of Hard Spheres to Silicate Liquids}

Despite the $\sim 70$ year history of the hard sphere model (Percus and Yevick, 1958), hard sphere theory is rather new to the study of silicate melts. The first notable application to geologic systems was presented by Guillot and Sarda (2006), where the compression of MORB, silica, and olivine composition liquids up to $10 \mathrm{GPa}$ were modeled to investigate high-pressure noble gas solubility. The effective hard sphere sizes were determined empirically by fitting 1 bar melt density measurements.

More recently, Jing and Karato (2011) calibrated oxide-specific hard spheres using liquid density and bulk modulus measurements in the $\mathrm{CaO}-\mathrm{MgO}-\mathrm{Al}_{2} \mathrm{O}_{3}-\mathrm{SiO}_{2}-$ $\mathrm{FeO}$ system. With least-squares minimization, they obtained best-fit HS diameters for each oxide component. Further refinements incorporated empirical corrections for temperature- and volume-dependence of the sphere sizes, but the absence of any physical model for these corrections limits their predictive power for out-of-sample data. As pointed out by the authors, these limitations are evident in the non-physical values for some of these terms (e.g. the sign of the temperature dependence for $\mathrm{MgO}$ spheres).

Despite the limitations of the empirical procedure employed by both Jing and Karato (2011) and Guillot and Sarda (2006), these studies demonstrated the potential power of the hard sphere model for describing pressure-, temperature-, and compositiondependent trends for oxide and silicate melt properties. These investigators used the HS model as a physically-motivated averaging kernel, allowing them to interpolate 
within the fitted dataset. Extrapolation was fairly limited, however, as the hard spheres were not strongly connected with physically meaningful aspects of liquid structure, thus confining it to pressures generally below $\sim 20 \mathrm{GPa}$. Compositionally-induced structure changes, such as the Ca-dependent Fe-coordination evolution inferred in Guo et al. (2013), are also inaccessible to this empirical approach. Motivated by both the successes and shortcomings of these earlier works, we extend the hard sphere modeling framework to directly incorporate the general structural attributes of dense oxidesilicate melts.

\section{Coordinated Hard Sphere Mixture (CHaSM)}

Evidence from diffraction studies, NMR spectroscopy, and FPMD simulations all indicate that cation coordination evolution is a common compression mechanism across all oxide and silicate melts. Despite this fact, there has been almost no development of simple theories for oxide melts that account for these coordination effects (a notable exception is the empirical thermodynamic modeling of amorphous $\mathrm{SiO}_{2}$ by Ghiorso (2004)). We thus develop the Coordinated Hard Sphere Mixture model (CHaSM), adding coordination-dependent structural and vibrational perturbations to the variational hard sphere model. Using the analytic formulation of the HS mixture model, CHaSM predicts the compression of silicate and oxide melts by determining the evolution of the cation coordination populations while remaining computationally efficient, needing only minutes on a standard desktop computer. Following a classical approach, CHaSM uses empirical potentials trained on the well-understood behavior of solid polymorphs, giving a fully predictive model for oxide liquids.

\subsection{Connecting Hard Spheres directly to Oxide Liquid Structure}

Previous applications of hard spheres to silicate systems suffered from their ambiguous link to atomic-scale melt structure. Since these melts are composed of charged ions with strong multi-body interactions, it is impractical to represent individual ions as hard spheres. Previous investigators side-stepped this complication, using the HS method as an empirical fitting function without providing physical justification for the extension from simple liquids to ionic melts. 
a

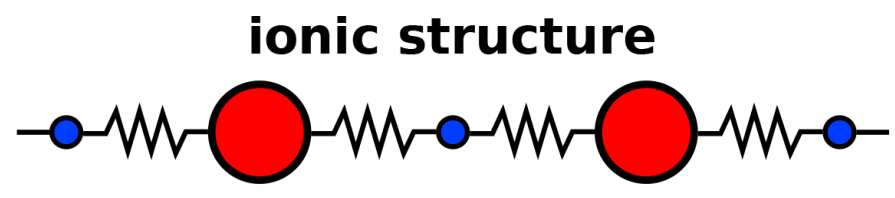

b ionic cluster model

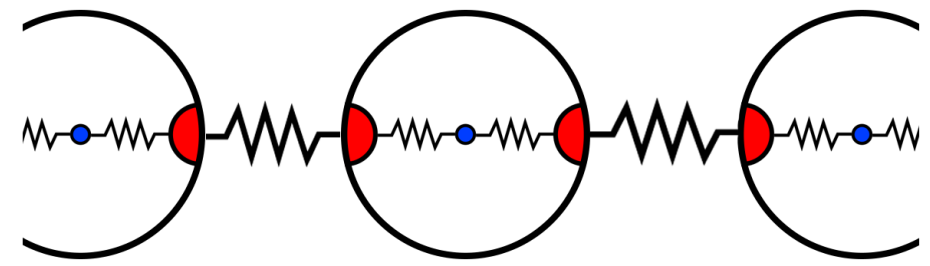

C coordinated ionic clusters

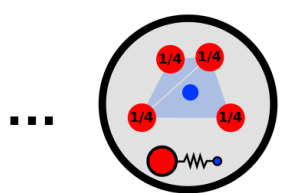

4

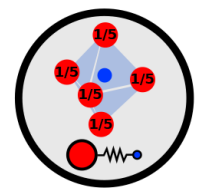

5

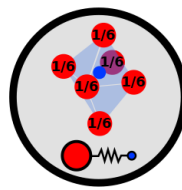

6

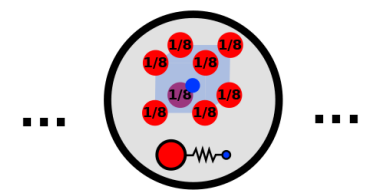

8

\section{CN}

Figure 2: Cartoon of oxide liquids and solids using charge-neutral ionic clusters. Panel (a) schematically represents a one-dimensional pseudo-lattice of oxygens (in red) and cations (in blue) with bonds represented by black springs. Panel (b) portrays the structure using ionic clusters, each containing a single cation and shared oxygens. Internal vibrational motions are represented by small black springs and cluster interactions are represented by the large black springs. Panel (c) represents the coordination-dependence of both internal vibrations and cluster interactions, where oxygen sharing is represented by fractional symbols.

To construct a physically-rooted hard sphere model, we consider a simplified cartoon of solid or melt structure as a set of coordinated ionic clusters, depicted in Figure 2. Panel (a) shows the condensed material as a pseudo-lattice of bonded cations and oxygens. These ions can be grouped, much like molecules in a gas, yielding the set of charge-neutral ionic clusters shown in panel (b). Each clustered particle is centered on a single cation, with shared oxygens between neighboring clusters. As with 
polyatomic gas molecules, we must account for the internal vibrational modes of each cluster (shown by small internal springs), in addition to cluster-cluster interactions (represented by the larger springs). Internal oscillators are used to account for the additional entropy and free energy associated with the relative motions of bonded ions. Finally, we recognize that the both cluster interactions and internal oscillations can depend on coordination number, as depicted in panel (c).

This conceptual structural model is justified both experimentally and theoretically by the radial distribution functions of silicate liquids, which universally show primary peaks corresponding to well-defined cation-oxygen bond lengths and average coordination numbers (for reviews, see Mysen and Richet 2005 and Karki 2015). Furthermore, the NMR work of (Stebbins et al., 1985) shows that the atomic structure of oxide and silicate melts is fully dynamic, with bonds constantly breaking and reforming, even at equilibrium. Each cations evolves through a set of coordination states, where the relative time spent in each state reflects the coordination populations.

\subsection{CHaSM Free Energy Contributions}

Since coordinated ionic clusters represent roughly independent structural units, they are perfectly suited for direct representation using hard spheres. The total Helmholtz free energy of the CHaSM liquid is thus given:

$$
F^{\mathrm{Tot}} \approx F^{\mathrm{HS}}+E^{\mathrm{struc}}+F^{\mathrm{osc}}
$$

where $F^{\mathrm{HS}}$ is the standard hard sphere mixture model term, $E^{\text {struc }}$ is the structural contribution to the internal energy, and $F^{\text {osc }}$ captures the internal oscillation contribution to each ionic cluster, which depend on the vibrational properties of both the individual bonded ions and the ionic clusters. As depicted in Figure 2, coordination number affects both the structural energy (through the cluster interactions) and the internal oscillations. In the next section, we present a method for estimating the potential parameters using the thermodynamic properties of solid crystals, and thus we assume for the current discussion that their values are known. (The full expressions for each of these terms are presented in Appendices A-E.) Given the dependencies of each term, 


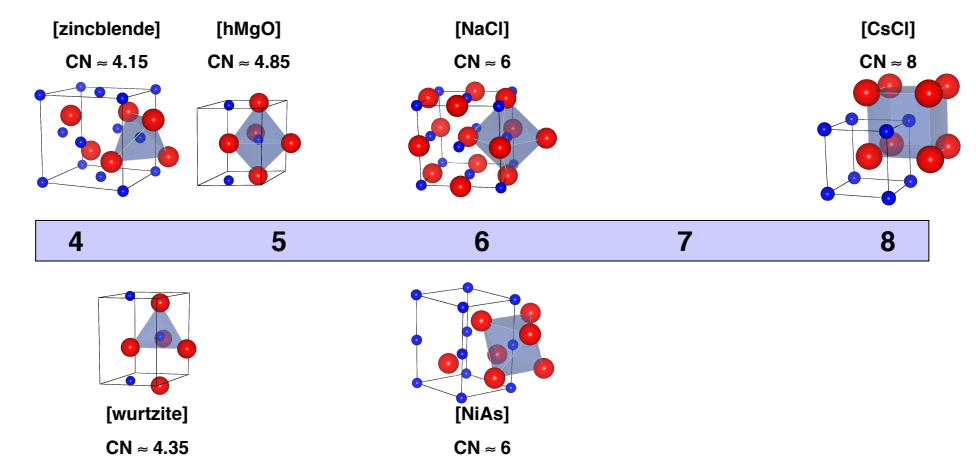

Figure 3: Unit cell structures and cation coordination polyhedra for six polymorphs of $\mathrm{MgO}$ displayed along a coordination spectrum (created using VESTA, Momma and Izumi, 2011). Though only the $\mathrm{NaCl}$ structure (periclase) is stable under terrestrial conditions, all structures can be theoretically can be studied and provide insight into the coordination-dependence of the clusters potentials for $\mathrm{MgO}$.

the total free energy can be written:

$$
F^{\mathrm{Tot}}=F^{\mathrm{Tot}}\left(V, T, \vec{X}, \mathbf{d}_{\mathbf{H S}}, \mathbf{p}_{\mathbf{H S}}\right)
$$

where the free energy is a function of the thermodynamic state variables volume $V$, temperature $T$, and composition $\vec{X}$, as well as the Hard Sphere diameters $\mathbf{d}_{\mathbf{H S}}$ and populations $\mathbf{p}_{\mathbf{H S}}$ specific to each coordination number and oxide component.

Using an extended form of the variational approach to Hard Spheres, discussed in Section 2.2, the optimal hard sphere diameters and coordination populations are determined using free energy minimization:

$$
F^{\mathrm{liq}}(V, T, \vec{X})=\min _{\mathbf{p}_{\mathrm{HS}}, \mathbf{d}_{\mathrm{HS}}}\left\{F^{\mathrm{Tot}}\right\}
$$

where this expression is analogous to Equation 3 for an evolving mixture of hard spheres. Due to its analytic underpinnings, this expression is rapidly evaluated enabling numerical minimization, predicting both coordination populations and total free energy.

\section{A First Application to MgO Melt}

As a first demonstration, we apply $\mathrm{CHaSM}$ to the $\mathrm{MgO}$ system, which is relatively simple yet vital to our understanding of the Earth. $\mathrm{MgO}$ exists as the mineral periclase 
(in the $\mathrm{B} 1$ or $\mathrm{NaCl}$ structure) over a large pressure range extending up to 400-500 $\mathrm{GPa}$, where it is predicted to transform to the denser $\mathrm{CsCl}$ (or B2) structure (Karki, 1997; Schleife et al., 2006; Jaffe et al., 2000; Joshi et al., 2012). Despite such stable behavior, other analog $\mathrm{AB}$ crystal systems indicate that $\mathrm{MgO}$ could potentially adopt a range of structures including the distorted NiAs structure and the low-coordination h-MgO (a buckled boron nitride structure), zincblende (B3), and wurtzite (B4) structures. Ab initio studies of these $\mathrm{MgO}$ polymorphs provide useful information about the coordination-dependence of atomic interactions in this system.

\subsection{Training Potentials on Solid Structures}

It is standard practice to use sets of well-characterized crystal structures to train ionic potential parameters, describing the interactions of cation-oxygen and oxygenoxygen pairs (e.g. Leinenweber and Navrotsky, 1988; Matsui, 1994, 1996; Gale, 1998; Guillot and Sator, 2007). Despite its successes, ionic potentials have demonstrated limited transferability to extreme near-endmember compositions (as shown by Guillot and Sator (2012) for very $\mathrm{FeO}$ - or $\mathrm{SiO}_{2}$-rich melts) or to very high pressures (as shown by Spera et al. (2011) for $\mathrm{MgSiO}_{3}$ melt at lower-mantle pressures). Both of these issues likely stem from the challenge of approximating ionic interactions over a wide range of local environmental conditions. It is unsurprising that simple analytic forms, such as the Buckingham or Morse potentials, are not flexible enough to represent coordination numbers that span from $\sim 4$ to $\sim 12$.

The development of accurate coordination-dependent ionic potentials would directly address this difficulty, but represents a substantial undertaking that deserves full attention in future studies. For CHaSM, we need only represent the dependence of total structural energy on cluster separation distances, and thus we adopt an empirical fitting approach to determine approximate ionic cluster potentials. Though more limited than coordination-dependent ionic potentials, it is much simpler to train the fewer free parameters and eliminates the need to explicitly include Coulombic interactions, as their energetic impact is folded into the pairwise interactions of the charge-neutral clusters.

For training our $\mathrm{MgO}$ cluster potentials, we rely on the dataset produced by Schleife 
et al. $(2006)^{2}$, which examined all of the available structures shown in Figure 3. The published energy-volume curves for the six potential structures of $\mathrm{MgO}$ are shown in Figure 4. Representing each crystal as a set of coordinated ionic clusters centered on the cation positions, we model the internal energy with a Morse potential (Equation (4)), summing over cluster pairs out to a cutoff distance large enough for convergence.

In addition to the direct interaction energy, we introduce a correction to account for the strong distortions present in some of the structures like $\mathrm{NiAs}$ and $\mathrm{hMgO}$. The cluster potentials developed here are appropriate for near-ideal atomic arrangements produced by regular packing schemes. Distorted structures have the freedom to adjust relative ionic positions, internal to the clusters, reducing the true energy cost of apparently unfavorable arrangements. To account for this discrepancy, we apply a simple additive energy offset correction:

$$
\Delta E_{\mathrm{corr}}^{0}=-\sum_{i} w_{i}\left(E_{\mathrm{pair}}\left(d_{\mathrm{Mg}-\mathrm{Mg}}^{i}\right)-E_{\text {pair }}\left(\left\langle d_{\mathrm{Mg}-\mathrm{Mg}}\right\rangle\right)\right)
$$

where $w_{i}$ is the weight of the $i^{\text {th }}$ nearest-neighbor cation at a distance $d_{\mathrm{Mg}-\mathrm{Mg}}^{i}$, and $\left\langle d_{\mathrm{Mg}-\mathrm{Mg}}\right\rangle$ is the average cation separation distance at zero pressure (see Appendix $\mathrm{F}$ ). This expression approximates the energetic benefit of shifting neighboring cations from their observed positions to their average separation distances, which better reflect the configuration's true energy cost. This corrective term is only significant for highly distorted structures like $\mathrm{NiAs}$ and $\mathrm{hMgO}$, where it is large and negative.

Parameter variations are fit in log-space to the $\mathrm{MgO}$ polymorph data assuming quadratic coordination-dependence:

$$
\delta \boldsymbol{\theta}_{\text {pot }}=\log _{e} \boldsymbol{\theta}_{\text {pot }}(\mathrm{CN})-\log _{e} \boldsymbol{\theta}_{\text {pot }}(6)=\boldsymbol{k}_{\mathbf{2}} \Delta \mathrm{CN}^{2}+\boldsymbol{k}_{\mathbf{1}} \Delta \mathrm{CN}
$$

where the fractional deviation of the potential parameters, $\delta \boldsymbol{\theta}_{\mathbf{p o t}}=\left(\delta r^{0}, \delta E^{0}, \delta \sigma\right)$, is expressed relative to their fitted values at $\mathrm{CN}=6$. The ambient pressure cation separation distances , $\log _{e} d_{\mathrm{Mg}-\mathrm{Mg}}(\mathrm{CN})$, also help to constrain the equilibrium position values, $\log _{e} r^{0}(\mathrm{CN})$, and are constrained to have the same slope and curvature $\left(k_{1}\right.$ and $\left.k_{2}\right)$ al-

${ }^{2}$ Self-consistent calculation of energies for all structures within a single study is crucial, since each study introduces an arbitrary energy offset. 


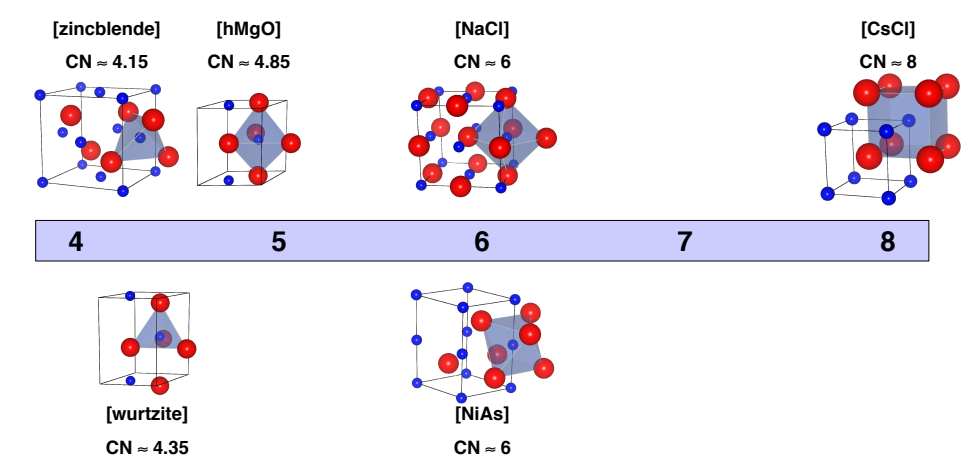

Figure 4: First-principles energy-volume curves for six solid polymorphs of $\mathrm{MgO}$ (Schleife et al., 2006), with the best-fit coordinated cluster potential model (panel a). Using 9 free parameters, plus an additional offset parameter, we obtain a good fit to the data with a goodness-of-fit (explained fraction of the variance) of $R^{2}=0.986$ (without the highly distorted NiAs structure; see text for details). Residuals are higher for more distorted structures, like NiAs and $\mathrm{hMgO}$, as expected. Panels (b) and (c) show the linear variation of relative bond-length and relative cation separation distance with coordination number, which reflect atomic packing efficiency.

Table 1: Best-fit Coordination-Dependent Morse Cluster Potential for $\mathrm{MgO}$

\begin{tabular}{llcc}
\hline & $\boldsymbol{\theta}_{\mathbf{p o t}}(6)$ & $\boldsymbol{k}_{\mathbf{2}}$ & \multicolumn{1}{c}{$\boldsymbol{k}_{\mathbf{1}}$} \\
\hline$r^{0}$ & $3.4359 \AA$ & +0.0045 & -0.0441 \\
$E^{0}$ & $0.6902 \mathrm{eV}$ & -0.0174 & +0.0088 \\
$\sigma$ & 0.2658 & -0.0010 & -0.0138 \\
\hline
\end{tabular}

Morse potential parameters (Equation 4) are assumed to vary quadratically with coordination number, as given by Equation (10).

lowing only a relative offset to freely vary, as shown by the dashed and solid red lines in Figure 5(a). An arbitrary energy offset parameter is also fit, reflecting that only relative energies are thermodynamically important.

We use standard least-squares regression to infer the coordination-dependent cluster potential for $\mathrm{MgO}$, excluding the NiAs dataset (see Table 1 for best-fit values). As can be seen in Figure 4(a), the NiAs structure deviates non-negligibly from the model curve, reflecting the limitations of the simple distortion correction term given by Equation 9. Furthermore, this structure is strongly disfavored as it forces all coordination 
polyhedra into energetically unfavorable distortions, and is only stable to small perturbations, indicating that a liquid with complete configurational freedom is unlikely to sample similar atomic geometries. Nevertheless, the model still provides a good qualitative match to the metastable NiAs curve, and the residuals to the other structures are all reasonable. . The goodness-of-fit can be quantified in terms of the explained fraction of the variance $R^{2}=1-\operatorname{Var}\left(E_{\text {resid }}\right) / \operatorname{Var}\left(E_{\text {data }}\right)$, with a value of 1 corresponding to a perfect fit. Excluding NiAs, the best-fit model gives a value of $R^{2}=0.99$ (which drops to $R^{2}=0.9$ if NiAs is included). Linear fits to normalized bond-lengths and cation separation distances, which both reflect atomic packing efficiency, are shown in panels b and $\mathrm{c}$ of Figure 4. The NiAs structure is the only outlier from these atomic packing trends, supporting its exclusion from the global fit. These quantities play an important role in the internal oscillation contribution to the free energy, as detailed in Appendix D.

The best-fit polynomials for the Morse potential parameters are shown in Figure 5 in terms of the fractional deviation from the fitted values at $\mathrm{CN}=6$. Panel (a) shows the systematic decrease of the equilibrium radius with increasing coordination, mirroring the $\mathrm{Mg}-\mathrm{Mg}$ distance trend, in red solid and dashed lines. Also shown are the variation of the energy-well depth and width, in gray and blue lines, respectively. In Panel (b), the effect of these trends on the predicted energy wells is shown, highlighting that the lowest equilibrium energy occurs for $\mathrm{CN}=6$, reflecting the strong stability of periclase. From this figure, we can also see that the minimum energy coordination state, given by the lowest energy curve at each distance, evolves upward with compression, revealing the primary driver for compression-induced coordination evolution in oxide liquids.

\subsection{Determining Liquid Properties using CHaSM}

Now that we can evaluate structural energies using the potentials obtained above, we apply CHaSM to determine average melt structure and thermodynamics. In this section, we demonstrate how to use the free energy minimization framework, introduced in Section 3.2, to calculate the thermodynamic properties of oxide liquids, specifically applying them to the $\mathrm{MgO}$ system.

We begin by representing a complex coordinated liquid in terms of a set of nearly- 

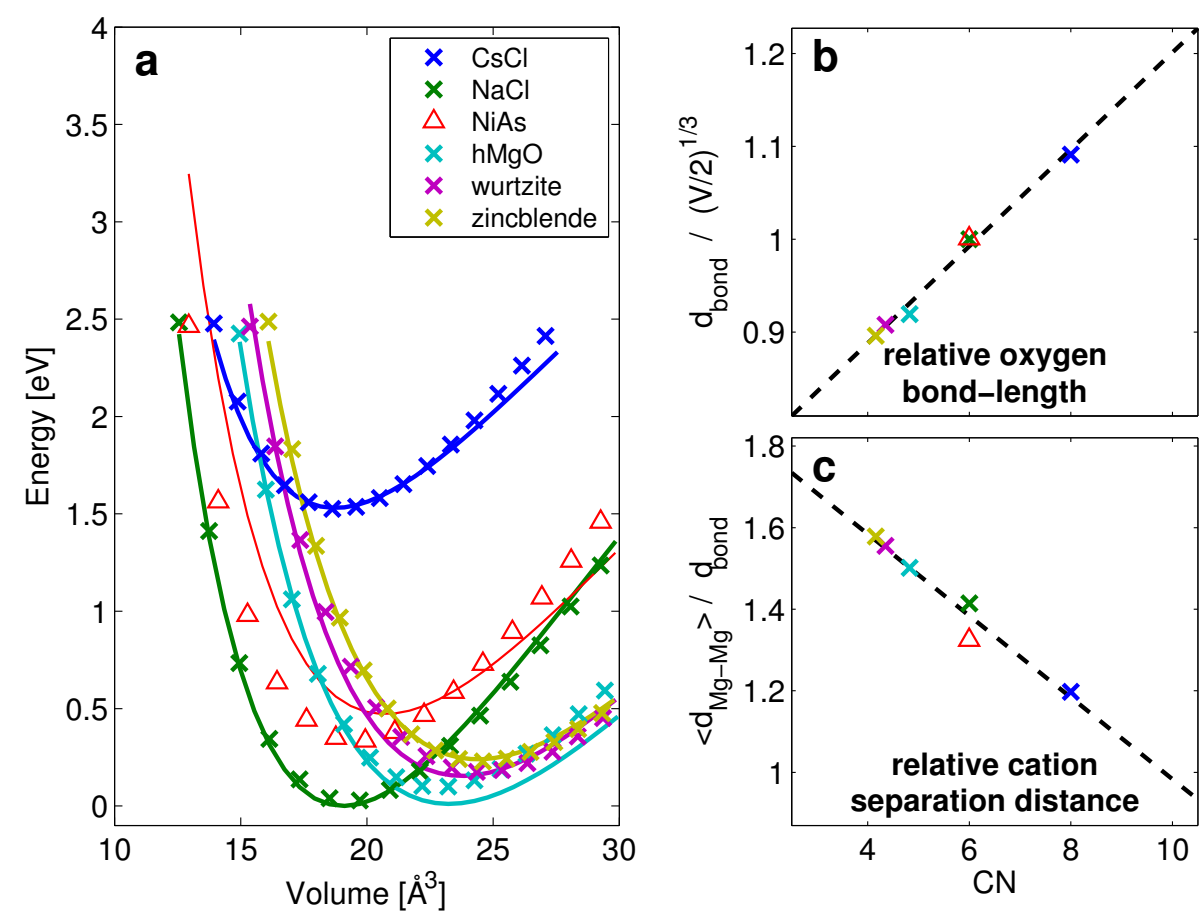

Figure 5: Best-fit dependence of cluster potential parameters on coordination number, in terms of fractional deviations from their reference value, $\delta \boldsymbol{\theta}_{\text {pot }}=\log _{e} \theta_{\text {pot }}(\mathrm{CN})-\log _{e} \boldsymbol{\theta}_{\mathbf{p o t}}(6)$. Panel (a) shows the variation of the position, depth, and width parameters in red, gray, and blue lines, respectively. Also plotted are the cation separation distances of the $\mathrm{MgO}$ polymorphs (symbols defined in Figure 4) and the corresponding fitted trend, shown in the red-dashed line, as discussed in the text. Panel (b) displays the corresponding potential energy wells, color-coded by coordination state.

independent cation coordination states. The essential insight behind this procedurethat coordination state occupancies can be treated as internal order parameters that are varied to minimize the total free energy-follows from the work of Ghiorso (2004). In that investigation, the author calibrated a thermodynamic model for $\mathrm{SiO}_{2}$ by empirically fitting observed coordination statistics from MD simulations of liquid and amorphous silica. The resulting model recovered evolving coordination populations, by modeling the energetic competition between volumetric work (akin to the structural energy) and the entropy of mixing. While this model is confined to pure silica and is limited by its need to train on liquid MD output, it demonstrates the explanatory power of the independent coordination-state assumption. Single-component liquids are thus 
viewed as a set of ionic clusters whose behavior changes gradually with coordination. Heterogeneous interactions (e.g. between 4- and 5-coordinated $\mathrm{Mg}$ ) are thus rather similar to homogeneous ones, and the proportion of heterogeneous interactions is limited by the peaked coordination distributions. Given coordination state independence, we are allowed to consider each state in isolation, greatly simplifying the calculation.

Using the variational method, we determine the optimal hard sphere size for each fixed-coordination liquid by separately minimizing its total free energy. Though coordination states are discretized, the structural energy of each state is not truly given by a single value as represented by this model, but rather a distribution of values reflecting continuous distortions of bonding polyhedra. Just as for crystal polymorphs, where fractional coordination numbers represent distortions from ideal bonding geometries, we approximate distorted states by considering non-integer values of the coordination number. This choice smooths over unphysical kinks in the energy surface that otherwise arise (a sampling frequency of 0.25 is sufficient for a converged result). Using standard optimization routines (available in MATLAB ${ }^{\circledR}$ ) to minimize the free energy, we determine the set of coordination-specific free energy curves for $3000 \mathrm{~K}$ MgO shown in Figure 6(a). Panel (b) shows the same free energy curves with the minimum energy envelope subtracted off, drawing attention to the compression-induced increase of the preferred coordination state from $\sim 4.5$ at $30 \AA^{3}$ to $\sim 8$ at $10 \AA^{3}$.

Treating these fixed-coordination liquids as a set of non-interacting states, we use Boltzmann's factors to estimate the equilibrium population distribution:

$$
p_{i} \propto \exp \left(-\Delta F_{i} / k_{B} T\right)
$$

where $\Delta F_{i}$ is the free energy difference from the minimum, $k_{B} T$ is the thermal energy scale, and $p_{i}$ is the population of the $i^{\text {th }}$ coordination state ${ }^{3}$. By examining the energetic spread of coordination states relative to the thermal energy scale $k_{B} T$ (shown by the redshaded region), we can roughly determine which states are well-populated, yielding a coordination spread of \pm 1 at $3000 \mathrm{~K}$.

\footnotetext{
${ }^{3}$ This equation is defined in terms of free energies $F_{i}$, instead of the internal energies $E_{i}$, to account for entropy variations, because the coordination states are degenerate macrostates rather than true microstates.
} 

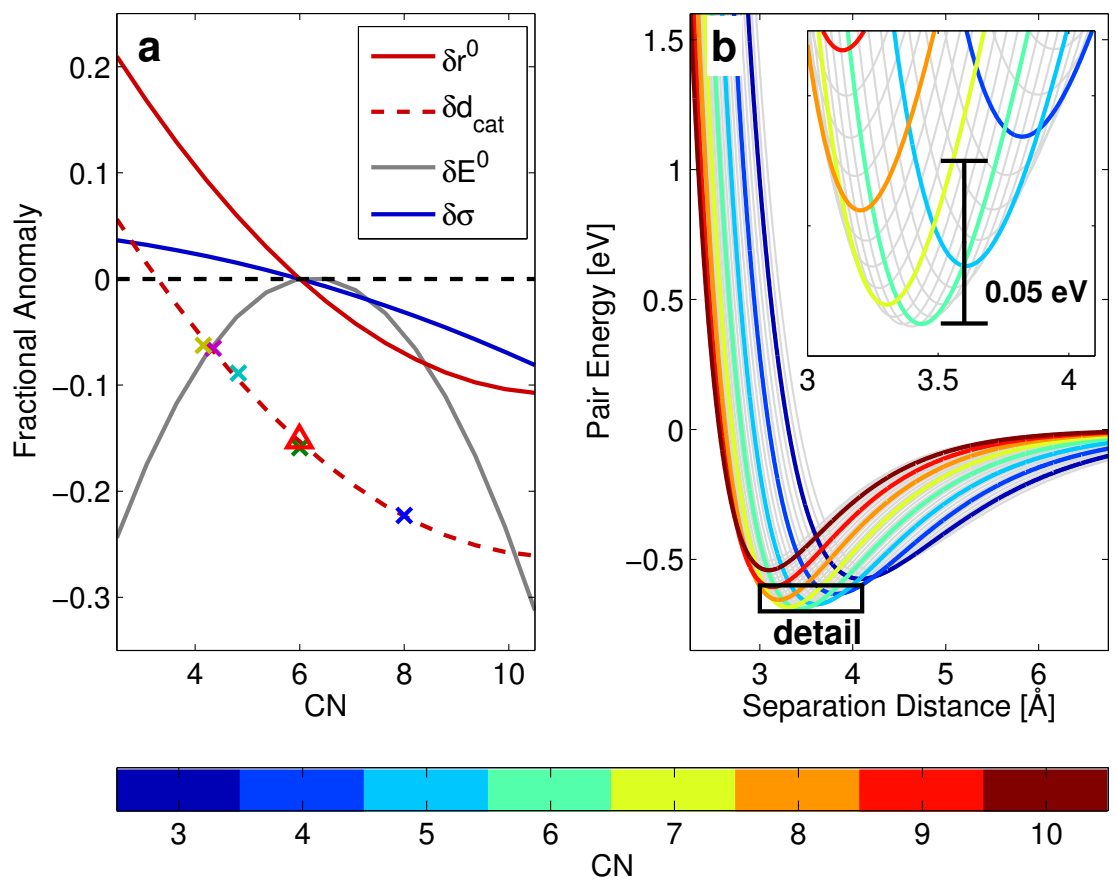

Figure 6: Free energy compression curves for hypothetical fixed-coordination $\mathrm{MgO}$ melts at $3000 \mathrm{~K}$, with cation coordination number ranging between 3 and 9; for visual clarity, only integer coordination states are color-coded (panel a). To emphasize the evolution of preferred coordination state with compression, the minimum energy envelope is removed in panel (b), showing steady increase in the dominant coordination state from 4 to 8 over the calculated volume range. The red shaded region indicates the thermal energy scale, $k_{B} T$, which determines the range of well populated coordination states about the dominant value (about \pm 1 ).

The coordination population distributions for $\mathrm{MgO}$ at $3000 \mathrm{~K}$, obtained by applying Equation (11) to the relative free energies, are shown in Figure 7a. Panel (b) shows the evolving median coordination state, with the $68 \%$ and $95 \%$ population intervals in the solid line, and dark and light gray bands, respectively, displaying the smooth upward trend of the average coordination number with compression. Also shown in red is the corresponding evolution at $7000 \mathrm{~K}$, where higher temperatures broaden the population distributions and shift the peak to higher coordination numbers, reflecting the drive toward more compact liquid particles, as shown for the toy-model in Figure 1d.

We repeat this procedure over a grid of points in volume-temperature space, to obtain the coordination populations over a range of conditions. The total free en- 


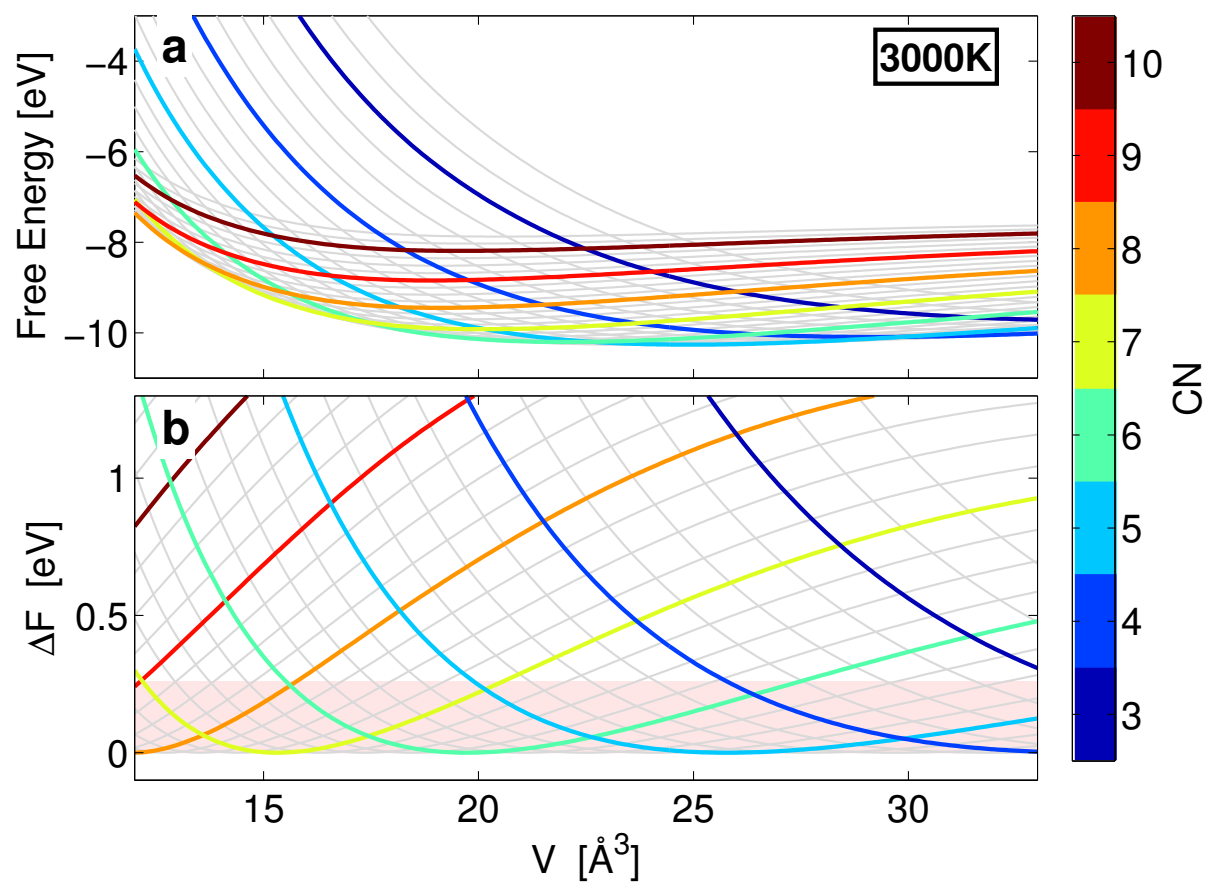

Figure 7: Evolution of the coordination populations for $\mathrm{MgO}$ melt at $3000 \mathrm{~K}$. Panel (a) shows colorcoded curves for the relative populations of each coordination state (with bins centered on integer values), and (b) shows the continuous evolution of coordination with compression, where the median, $68 \%$, and 95\% population intervals are indicated by the black line, dark gray, and light gray bands, respectively. For comparison, the median and $68 \%$ population bounds are also shown for $7000 \mathrm{~K}$, in the solid and dashed red lines.

ergy is given by a weighted average of the independent contributions from each coordination state combined with the free energy of mixing term from Appendix A, $F_{\text {liq }}=\sum_{i} p_{i} F_{i}+F^{\text {mix }}$. Each equilibrium configuration is calculated in a few seconds, and the entire range of conditions is finely sampled in minutes.

To determine all of the thermodynamic properties of interest, we must represent these samples with a smooth free energy surface appropriate for calculating successive temperature and volume derivatives. We use a $\log$-EOS, which is simply a Taylor expansion in logarithmic strain, defined as $\epsilon \equiv \log _{e}\left(V / V_{r}\right)$, where $V_{r}$ is a convenient reference volume. Unlike other EOS forms, the log-EOS function retains a polynomial form even if the zero-pressure volume is not known a-priori, allowing basic polynomial 


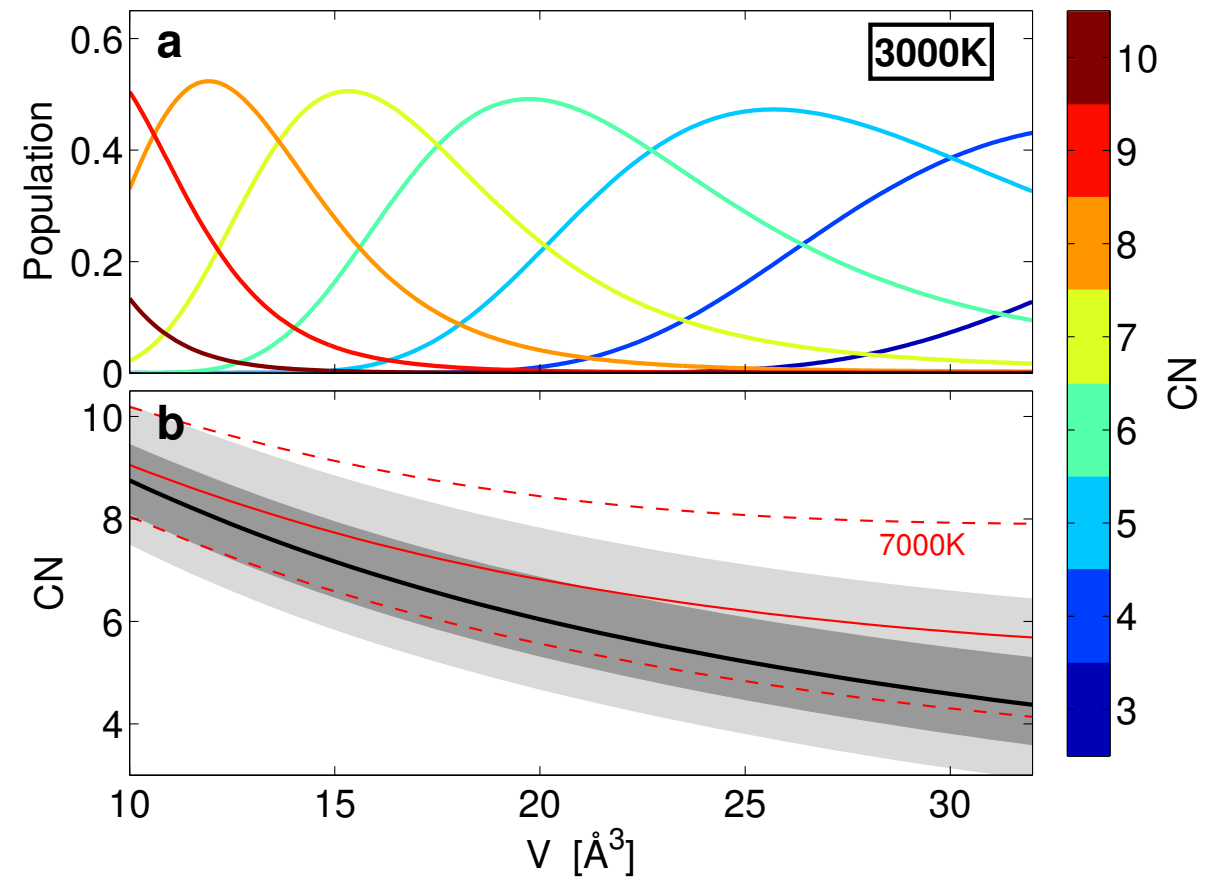

Figure 8: Derivative equation of state properties, pressure and entropy, for $\mathrm{MgO}$ melt. The main panel shows the pressure-volume isotherms color-coded by temperature, which agree well with first-principles results from Karki et al. (2006) at $3000 \mathrm{~K}$ and $7000 \mathrm{~K}$, shown in the blue and red crosses, respectively. The inset figure displays the entropy-compression evolution, where the blue cross corresponds to the ambient pressure entropy of 10.5 per atom from de Koker and Stixrude (2009), which should be compared with the value of 9.8 indicated by the open circle. The reference volume of $V_{10}^{\text {ref }}=20.2 \AA^{3}$, defined at $10 \mathrm{GPa}$, accounts for a systematic scaling offset of the CHaSM results and should be compared to the first principles value of $22.5 \AA^{3}$.

regression for inferring the coefficients of each free energy isotherm. We find that a $4^{\text {th }}$ order expansion is fully sufficient to explain the variation to within the precision of the model. Finally, we capture the temperature dependence of the coefficients using spline interpolation. This straightforward method provides a complete description of the free energy surface that is thermodynamically consistent and smooth up to the highest-order derivatives of interest.

Given the full free energy surface for $\mathrm{MgO}$ melt, we obtain the isothermal pressure and entropy curves through differentiation shown in Figure 8. Also plotted in blue and 
red crosses are the first principles calculated values from Karki et al. (2006) for MgO at $3000 \mathrm{~K}$ and $7000 \mathrm{~K}$, respectively. CHaSM's agreement with this simulation is quite remarkable, given the $>4-5$ orders of magnitude difference in computational resources for these methods. This comparison uses scaled volumes, as is common practice for theoretical calculations, since even first principles MD simulations produce systematic errors in reference volumes of $\sim 6-13 \%$, as estimated from the typical $\sim 2$ GPa empirical pressure correction (Van de Walle and Ceder, 1999; Oganov et al., 2001; Stixrude and Karki, 2005) applied to silicate melts with reference bulk moduli of $\sim 15-30 \mathrm{GPa}$ (Jing and Karato, 2011)). Given the simplicity of CHaSM and the approximate nature of the cluster potentials utilized in this work, we are quite satisfied with a volumetric difference of $\sim 9 \%$ for a reference pressure of $10 \mathrm{GPa}$. We can also see in the inset figure that the $3000 \mathrm{~K}$ ambient pressure entropy predicted by CHaSM matches the first principles value to within $\sim 6 \%$ (de Koker and Stixrude, 2009). This level of agreement in terms of entropy stems from CHaSM's accounting for the internal oscillations of each ionic cluster, in contrast to simpler hard sphere approaches that all drastically underestimate the entropy.

\section{Understanding the Grüneisen Parameter Evolution of Liquids}

As discussed in the introduction, the grüneisen parameter determines the slope of the adiabat, governing the thermal profiles in convecting systems (see Equation 1). Its thermodynamic definition is:

$$
\gamma \equiv-\left.\frac{\partial \log _{e} T}{\partial \log _{e} V}\right|_{S}=\frac{\alpha K_{T} V}{C_{V}}
$$

where $\alpha$ is the thermal expansion, $K_{T}$ is the isothermal bulk modulus, and $C_{V}$ is the heat capacity at constant volume. Using this equation, we evaluate the compression evolution of the grüneisen parameter for a set of isotherms for $\mathrm{MgO}$ melt, shown in Figure 9. The increase with compression, opposite that of solids, is nearly universal for liquids. The magnitude of the increase is in reasonable agreement with first principles calculations, though our values are systematically lower by $\sim 0.1$ to 0.2 (de Koker and Stixrude, 2009). We also find a substantial temperature dependence for $\gamma$, another 


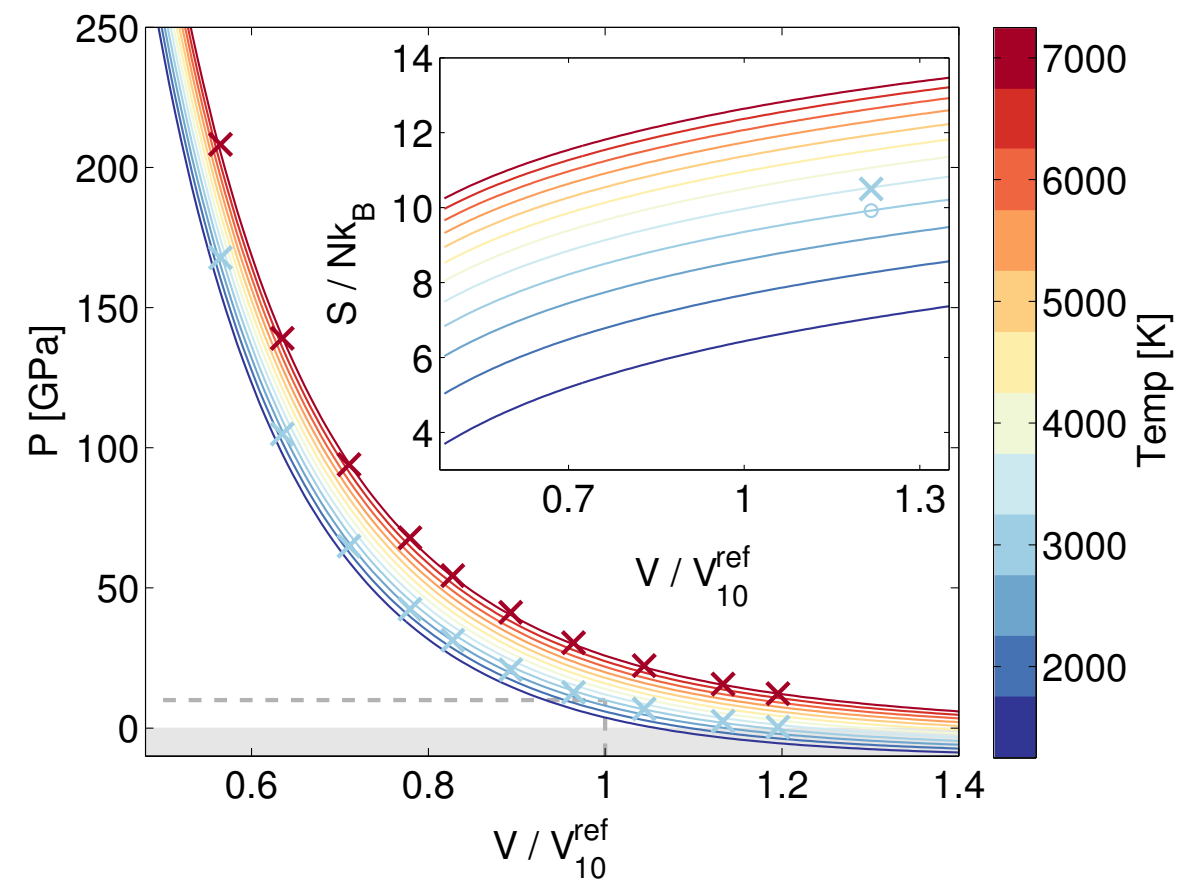

Figure 9: The thermodynamic grüneisen parameter $(\gamma)$ of $\mathrm{MgO}$ melt, predicted by $\mathrm{CHaSM}$, is plotted for a set of color-coded isotherms, displaying the universal increasing trend with compression for liquids. Also plotted are the mode grüneisen parameters at $3000 \mathrm{~K}$ for the ionic cluster and internal oscillator vibrations, shown in circles and triangles, respectively (determined using Equations 13 and 15). The weighted average of these two vibrational modes yields the total vibrational grüneisen parameter, shown in blue crosses, which provides an excellent match to the thermodynamic trend at $3000 \mathrm{~K}$.

distinguishing general feature of liquids. CHaSM predicts that $\gamma$ and temperature are positively correlated for $\mathrm{MgO}$ melt, while first principles investigation obtained a dependence of similar magnitude but opposite sign (de Koker and Stixrude, 2009). It is unclear, however, whether this disagreement is a result of the MD simulations themselves, or rather of the parametric modeling needed to predict $\gamma(V, T)$ from a limited set of MD simulation runs affected by statistical noise.

The widespread paradoxical $\gamma$-behavior of liquids was first remarked upon by Knopoff and Shapiro (1970), who observed that the grüneisen parameter of water increases consistently for volumetric compressions of up to $20 \%$. They also found a strong positive correlation with temperature, much like our findings for $\mathrm{MgO}$, in contrast to the near 
temperature-independent behavior of solids. Since then, similar behavior has been seen for a wide array of liquids, including: cryogenic Ar; organic solvents like pentane, ethanol and methanol; metallic liquids like mercury; and a variety of silicates in the CMASF compositional system (Brown et al., 1987; Amoros et al., 1988; Boehler and Ramakrishnan, 1980; Asimow, 2012).

Observed for nearly every liquid type-including neutral atomic, molecular, metallic, and partially ionic silicate liquids - the grüneisen behavior of liquids requires a general explanation befitting its broad insensitivity to chemistry. From statistical physics, it is easy to show that increasing $\gamma$ is a natural outcome of the basic hard sphere model, as noted by Jing and Karato (2011), resulting from the evolution of the packing fraction $\eta$ with increasing compression. Unfortunately, this result cannot be quantitatively generalized to multi-component or highly compressed liquids.

Within the Earth science community, the currently favored explanation rests on an analogy to the behavior of solid $\mathrm{MgSiO}_{3}$ polymorphs, which individually show decreasing $\gamma$-trends, but between which there is a systematic upward shift with increasing coordination number. Since a compressing liquid effectively samples ionic clusters that are similar to increasingly higher pressure polymorphs, we might expect a corresponding increasing trend in $\gamma$ (Stixrude and Karki, 2005). Like the basic hard sphere behavior, this cation coordination model revolves around structural evolution, but it also fails to generalize since most non-oxide liquids do not undergo cation coordination evolution. Even for oxide or silicate melts, it does not provide testable quantitative estimates, as it relies on a qualitative interpolation argument. This uncertainty is most notable for molten silica with $\gamma=0$ at ambient pressure(Asimow, 2012), in contrast to its low-pressure solid polymorphs which show a wide-range of values of roughly $0,0.3$, and 0.6 for $\alpha$-quartz, coesite, and $\beta$-quartz, respectively, despite all possessing four-coordinated silica tetrahedra(Boehler, 1982; Hemley, 1987; Williams et al., 1993; Welche et al., 1998).

To calibrate our intuition, we review the well-understood behavior of crystalline solids where the entropy is dominated by atomic vibrations. The thermodynamic grüneisen parameter (Equation 12) can be directly interpreted in terms of the volumedependence of the angular frequency $\omega$ of each vibrational mode in the crystal (Grüneisen, 
1912, 1926):

$$
\gamma_{i}=-\frac{\partial \log _{e} \omega_{i}}{\partial \log _{e} V}=-\frac{1}{2} \frac{V}{\omega_{i}^{2}} \frac{\partial \omega_{i}^{2}}{\partial V}
$$

where $\gamma_{i}$ is the mode-specific grüneisen parameter, and the total thermodynamic value can be shown to be a weighted average of the individual mode contributions,

$$
\gamma=\frac{\sum C_{V, i} \gamma_{i}}{\sum C_{V, i}}
$$

with a weights equal to the mode-specific heat capacity, $C_{V, i}$ (e.g. Kieffer, 1976).

To better understand the vibrational definition of $\gamma$, we introduce an approximate expression for the particle vibrational frequency, representing independent oscillation of each particle about its average position:

$$
\begin{gathered}
m \omega^{2}=\hat{k}_{\mathrm{tot}}=\nabla^{2} E_{\mathrm{struc}} \approx \sum_{j} N_{j} \hat{k}_{j} \\
\hat{k}_{j}=\frac{1}{6}\left[E_{\mathrm{pot}}^{\prime \prime}\left(r_{j}, \boldsymbol{\theta}_{\mathrm{pot}}\right)+\frac{2}{r_{j}} E_{\mathrm{pot}}^{\prime}\left(r_{j}, \boldsymbol{\theta}_{\mathrm{pot}}\right)\right]
\end{gathered}
$$

where $\hat{k}_{\mathrm{tot}}$ is the total restoring force constant of each particle and $\hat{k}_{j}$ represents the contribution by interactions with the $N_{j}$ particles occupying the $j^{\text {th }}$ particle distance. This expression is obtained by taking a second derivative of the structural energy with respect to perturbations in particle position (see for example Roy et al., 1974) and also forms the basis of the internal oscillator correction within CHaSM (see Appendix D).

This expression provides a crude representation of the shortest wavelength phonons in a material, akin to the Einstein model, providing useful semi-quantitative insights for solid materials. First, we can see that the temperature-independence of the grüneisen parameter (e.g. Soga and Anderson, 1966) should arise for nearly-harmonic vibrations, where the force constant is independent of amplitude, as assumed by the quasiharmonic model (e.g. Gale and Rohl, 2003). It is also well established from both experiments and theory that $\gamma$ always decreases with compression for solid phases (e.g. McQueen and Marsh, 1960; Anderson, 1968; Roberts and Ruppin, 1971; Boehler, 1982).

The first investigation to examine the application of these ideas to liquids, (Knopoff and Shapiro, 1970), put forward its own simple semi-quantitative model, appealing to 
the development of progressively larger solid-like clusters within the liquid as it is compressed. The authors showed that for a 1D monatomic crystal, the growth of solid-like clusters would generate the observed behavior as the quantity and value of vibrational mode frequencies evolved with increasing cluster size. This explanation using local vibrations in a liquid is attractive due to its generality, but the over-simplified derivation lacks quantitative predictive ability for real complex liquids.

In contrast to solids, where the average particle distances are defined uniquely by the crystal structure and the volume, liquids contain typical particle separations corresponding to successive shells of neighboring particles. The sizes and occupancies of these neighboring shells evolve with compression, reflecting the development of shortrange order as expressed in the radial distribution function (see Appendix D). When applied to liquids, this approximation becomes paradoxically more accurate, since the long-wavelength phonons that it neglects are largely absent from liquid dynamics. Particle trajectories within a liquid are stochastic and complex, combining both oscillatory and diffusive motions (e.g., Angelani and Foffi, 2007; Trachenko and Brazhkin, 2013). We therefore interpret Equation (15) as an approximate expression for the vibrational component of motion experienced by each particle in response to interactions with the time-averaged liquid structure surrounding it.

As $\mathrm{CHaSM}$ enables us to calculate the frequencies of these motions, we can directly test whether the solid-derived theories for the grüneisen parameter can be extended to liquids. We thus calculate the effective mode grüneisen parameters (Equation 13) for CHaSM's ionic-cluster and internal oscillator vibrations using the complete expressions for vibrational frequencies in Appendix D, as shown by the open symbols in Figure 9. The internal oscillator vibration represents the independent relative motions of the bonded $\mathrm{Mg}$ and $\mathrm{O}$ ions, and the cluster vibration represents the motions of each coordinated ionic cluster relative to its environment (where bonded $\mathrm{Mg}$ and $\mathrm{O}$ ions move roughly in-phase with one another). Using the standard heat capacity weighted average, we combine these two contributions into the total vibrational grüneisen parameter, shown in the blue crosses, which yields an excellent match to the thermodynamically determined values in the solid blue line. Unlike for nearly-harmonic solids, the vibrational frequencies determined by CHaSM are functions of both volume and 
temperature, $\omega(V, T)$, reflecting how thermally-induced structural evolution alters the average atomic environment, influencing restoring forces.

Determining the average vibrational $\gamma$ value from the local vibrational modes within a liquid clearly yields a predictive quantitative model for its thermodynamic grüneisen parameter. Behavioral differences between liquids and solids can thus be understood by decomposing the possible sources of volume-dependence for atomic vibrations:

solids \& liquids

$$
\frac{d \omega^{2}}{d V} \approx \frac{\partial}{\partial V}\left(\sum_{j} N_{j} \hat{k}_{j}\right) \approx \quad+\underbrace{\sum_{j} N_{j} \frac{\partial \hat{k}_{j}}{\partial r_{j}}\left(\frac{d r_{j}}{d V}\right)_{\text {geom }}}_{\begin{array}{c}
\text { bond length } \\
\text { compression }
\end{array}}
$$

\section{liquids only}

$$
+\underbrace{\sum_{j} N_{j} \frac{\partial \hat{k}_{j}}{\partial r_{j}}\left(\frac{d r_{j}}{d V}\right)_{\text {struc }}+\sum_{j} \frac{\partial N_{j}}{\partial V} \hat{k}_{j}}_{\begin{array}{c}
\text { short-range order } \\
\text { development }
\end{array}}+\underbrace{\sum_{j} N_{j} \frac{\partial \hat{k}_{j}}{\partial \boldsymbol{\theta}_{\text {pot }}} \frac{d \boldsymbol{\theta}_{\mathbf{p o t}}}{d V}}_{\begin{array}{c}
\text { environment-dependent } \\
\text { pot interactions }
\end{array}}
$$

where the potential parameter vector is represented by $\boldsymbol{\theta}_{\text {pot }}$. We can understand the qualitatively different $\gamma$-behavior of liquids and solids, related to vibrations through Equation 13, by considering the expected volume-dependence of each term in this expression.

In the case of solids, where atomic structures are constrained by symmetry, compression is accomplished predominately by bond-length reduction, represented by the first term in Equation (16). This leads to the normal solid-like compression behavior of decreasing $\gamma$, which is a direct consequence of the non-linear repulsive potential that dominates short-range interactions as demonstrated by Anderson (1968). Liquids, on the other hand, are able to dynamically adjust their structure in order to minimize free energy, compressing by a combination of structural relaxation, $\left(d r_{j} / d V\right)_{\text {struc }}$, and solidlike fixed-structure compression, $\left(d r_{j} / d V\right)_{\text {geom }}$. This corresponds directly in the hard sphere framework to an initial compression at fixed packing fraction-where the ratio of hard sphere volume to total volume is held constant - and then allowing the hard sphere size to expand to minimize total free energy. Due to short-range non-linear repulsive forces, the hard sphere size always grows during relaxation, reducing the large 
repulsive energy cost of closely spaced particles.

As liquid packing fraction increases to better accommodate compression, shortrange order further develops, increasing the number of nearest neighbors, given by $d N_{j} / d V$. The two short-range order terms in Equation (16) have competing effects on the vibrational frequency, but the exponential drop (due to increasing nearest neighbor distance, $r_{j}$ ) dominates over the linear rise (due to increasing shell occupancy, $N_{j}$ ). This net frequency drop with compression provides a negative contribution to the grüneisen parameter for liquids. The strength and duration of the effect are limited, however: as the liquid is compressed, its packing fraction rises reflecting a more compact and solid-like structure. The potential for further structural evolution is reduced, however, as the contrast between liquid and solid diminishes.

The final term in the vibrational frequency reflects the dependence of pairwise interactions on the local-environment. Within the CHaSM framework, this manifests as the cation coordination dependence of the cluster potentials, $\boldsymbol{\theta}_{\text {pot }}(\mathrm{CN})$. The compressioninduced evolution of cation coordination state provides a crucial structural degree of freedom that lowers the overall energy by significantly reducing the curvature of the free-energy surface. This decrease in material stiffness lowers the compression trend of the vibrational restoring force, thus providing a negative contribution to $\gamma$ whose magnitude falls with compression, similar to the short-range order contribution discussed above. This similarity is perfectly natural, since the bonding coordination-dependence of the cluster potentials is designed to capture the development of short-range order at atomic-bonding length-scales.

Given the opposing effects of the geometric and structural contributions to the grüneisen parameter, we can see that the liquid-like behavior of $\gamma$ should be limited to a material-specific compression range. Once the contrast between liquid and solid structures is sufficiently small, the negative contribution of the liquid-specific terms can no longer overwhelm the positive fixed-geometry term, and solid-like behavior will develop. This implies that liquids with close-packed structures, like molten iron, cannot access the necessary structural degrees of freedom and should thus display solid-like $\gamma$ trends under all P-T conditions, as supported by the experimentally observed structure (Shen et al., 2004) and theoretically calculated $\gamma$-trends of molten iron over a broad 
range of pressures (Vocadlo et al., 2003). In contrast, the room temperature grüneisen parameter of mercury, also a metallic liquid, shows the usual increasing trend up to its freezing point (Boehler and Ramakrishnan, 1980), as it evolves from an initial selfcoordination number of 6 at ambient pressure up to a close-packed structure near 12 at the freezing point (Kruh et al., 1963). We further expect that particularly open low density structures with low cation coordination numbers, such as liquid silica, should demonstrate markedly strong grüneisen parameter evolution, reflecting the large initial contrast between the liquid and solid structures. This matches the experimentally observed and theoretically calculated behavior of silica, which evolves from $\gamma=0$ at ambient pressure to $\gamma \sim 1$ at two-fold compression (Asimow, 2012; de Koker and Stixrude, 2009).

This model thus provides a simple explanation of why increasing grüneisen parameter upon compression does not occur in solid structures, but should be generally present up to some threshold pressure for nearly all liquids. The ability of liquids to adjust their structures in order to minimize free energy is a generic consequence of the lack of long-range order present in all liquids, and so it is natural to expect such universal behavior. Similar to compression, thermally-driven structural evolution in liquids also affects the packing efficiency and so imposes an analogous temperature dependence to vibrational frequencies. We therefore expect that liquids should generally exhibit temperature dependence of the grüneisen parameter, as first observed by Knopoff and Shapiro (1970).

\section{Discussion and Conclusions}

We present a new simplified model for representing the structure and thermodynamics of oxide and silicate melts. The Coordinated Hard Sphere Mixture (CHaSM) provides an extension to the standard hard sphere mixture model, which accounts for the evolution of local cation coordination number, a primary compression mechanism for oxide melts. Atoms in the liquid are represented as a set of neutrally-charged ionic clusters, each composed of a single cation surrounded by a shell of shared bonded oxygens. CHaSM is based on the principle of free energy minimization, where the 
sizes and populations of the coordinated ionic clusters (represented by hard spheres), are directly predicted by the model as a function of volume and temperature. Unlike previous adaptations of hard sphere theory to silicate melts, CHaSM accounts for the internal oscillations of bonded ions and so is able to accurately predict thermodynamic properties related to entropy, making it uniquely suited to the exploration of high P-T melt properties such as grüneisen parameter evolution.

As a first application, we demonstrate how the CHaSM framework accurately predicts the thermodynamic properties of $\mathrm{MgO}$ melt over a wide pressure-temperature range. Coordination-dependent interactions between clusters are described by potentials that are trained on data from solid polymorphs, which sample the energetics of structures over a wide range of coordination values. Using these potentials, CHaSM estimates coordination state evolution and equation of state properties that compare favorably with first principles calculations, despite the 4 to 5 order of magnitude difference in computation time. As a statistical physics model, CHaSM does not suffer from the convergence issues that confront MD simulations and thus extends naturally to near-solidus temperatures, enabling future comparison with laboratory measurements at experimentally attainable temperatures.

Finally we present a simple new approach to understanding grüneisen parameter evolution characteristics common to nearly all liquids. By considering pairwise interactions, typical vibrational frequencies are determined from the average liquid structure of $\mathrm{MgO}$ for ionic clusters and the bonded ions that comprise them. Analysis of different contributions to the particle restoring force shows why compression-induced growth in the grüneisen parameter is such a universal feature of liquids, reflecting their ability to evolve toward more compact and solid-like structures upon compression. By focusing only on independent particle motions, this approximation neglects any role played by longer wavelength vibrations. The close match between our local vibrational model and the calculated thermodynamic grüneisen parameter from CHaSM indicates that, despite its simplicity, this model accurately captures most of the important physics.

The next challenge for CHaSM is extension to a broad geologically interesting chemical system. This can be achieved while improving accuracy up to moderate pressures $(\lesssim 30 \mathrm{GPa})$ by implementing standard ionic potentials for modeling struc- 
tural energies. Similar to our approach for $\mathrm{MgO}$ polymorphs in this investigation, we can identify coordination-dependent structural trends, like those in Figure 4, across a broad collection of minerals throughout a geologically relevant chemical system like $\mathrm{CaO}-\mathrm{MgO}-\mathrm{Al}_{2} \mathrm{O}_{3}-\mathrm{SiO}_{2}-\mathrm{FeO}$. These trends in ionic pair distances reflect the influence of cation coordination on the atomic packing geometry. By initially limiting investigation to moderate pressures and intermediate compositions, we can make use of existing ionic potentials, like those of Matsui $(1994,1996)$ and Guillot and Sator (2007), while avoiding the transferability limits of the potentials induced by significant coordination evolution. The approximations of CHaSM could also be directly tested against empirical MD simulations using the same ionic potentials. As discussed in Section 4.1, the CHaSM framework would also greatly benefit from the development of coordination-dependent ionic potentials, enabling significantly more accurate calculations over broader ranges of chemistry and pressure. The training of such potentials should greatly expand the general reach of empirical atomic simulation techniques, though it is likely to require input from first-principles calculations on solid phases to constrain the greater number of free parameters.

Given that CHaSM provides a complete description of the free energy surface of the liquid, we will also be able to carry out melting curve calculations by combining it with the thermodynamic properties of the solid phases, allowing direct comparison with high-pressure melting experiments (e.g. Ohtani, 1983; Knittle et al., 1989; Boehler, 2000; Ohtani, 2009; Andrault et al., 2011). The results of such an investigation will also be interesting to compare with the recent first-principles work on the $\mathrm{MgO}-\mathrm{SiO}_{2}$ binary by de Koker et al. (2013).

As the CHaSM model is extended to a broad chemical system, we might also begin incorporating constraints from liquid data as well. This could be accomplished as a perturbation to the model parameters trained on solid structures, leading to solutions that are loosely guided by the solid-derived values, rather than fixed to them. Such an approach might eliminate the need for volumetric scaling, for instance, while improving the zero-pressure entropy values. Though we expect these sorts of implementation details to evolve over time, we are confident that CHaSM provides the necessary framework to produce accurate predictions of melt thermodynamics, as it remains tied to a 
simplified yet realistic physical picture of oxide and silicate melts.

\section{Acknowledgments}

The authors would like to thank Becky Lange, Youxue Zhang, Jean-Phillipe Harvey, Jennifer M. Jackson, and Jonathan Stebbins for useful conversations throughout the development of this study. We also would like to thank the NSF for supporting this work through awards EAR-1119522 and 1226270, and the Turner Postdoctoral

Fellowship at the University of Michigan.

\section{References}

Amoros, J., Solana, J., Villar, E., 1988. Temperature, pressure and volume dependence of the Grüneisen parameter of dense gaseous and liquid argon. Materials Chemistry and Physics 20, 255-260.

Anderson, O.L., 1968. Some remarks on the volume dependence of the Grüneisen parameter. J. Geophys. Res. 73, 5187-5194.

Andrault, D., Bolfan-Casanova, N., Nigro, G.L., Bouhifd, M.A., Garbarino, G., Mezouar, M., 2011. Solidus and liquidus profiles of chondritic mantle: Implication for melting of the Earth across its history. Earth and Planetary Science Letters 304, 251-259.

Angelani, L., Foffi, G., 2007. Configurational entropy of hard spheres. Journal of Physics: Condensed Matter 19, 256207-.

Asimow, P.D., 2012. Shock compression of preheated silicate liquids: Apparent universality of increasing Grüneisen parameter upon compression. AIP Conf. Proc. $1426,887-890$.

Boehler, R., 1982. Adiabats of quartz, coesite, olivine, and magnesium oxide to 50 kbar and $1000 \mathrm{~K}$, and the adiabatic gradient in the Earth's mantle. J. Geophys. Res. 87, 5501-5506. 
Boehler, R., 2000. High-pressure experiments and the phase diagram of lower mantle and core materials. Rev. Geophys. 38, 221-245.

Boehler, R., Kennedy, G.C., 1977. Pressure dependence of the thermodynamical Grneisen parameter of fluids. Journal of Applied Physics 48, 4183-4186.

Boehler, R., Ramakrishnan, J., 1980. Experimental results on the pressure dependence of the Grüneisen parameter: A review. J. Geophys. Res. 85, 6996-7002.

Brown, J.M., Furnish, M.D., Mcqueen, R.G., 1987. Thermodynamics for (Mg, Fe)2SiO4 from the Hugoniot, in: High-Pressure Research in Mineral Physics: A Volume in Honor of Syun-iti Akimoto. American Geophysical Union, pp. 373-384.

Funamori, N., Yamamoto, S., Yagi, T., Kikegawa, T., 2004. Exploratory studies of silicate melt structure at high pressures and temperatures by in situ X-ray diffraction. J. Geophys. Res. 109, B03203-.

Gale, J.D., 1998. Analytical Free Energy Minimization of Silica Polymorphs. J. Phys. Chem. B 102, 5423-5431.

Gale, J.D., Rohl, A.L., 2003. The General Utility Lattice Program (GULP). Molecular Simulation 29, 291-341.

Ghiorso, M.S., 2004. An equation of state for silicate melts. I. Formulation of a general model. American Journal of Science 304, 637-678.

Grüneisen, E., 1912. Theorie des festen Zustandes einatomiger Elemente. Ann. Phys. 344, 257-306.

Grüneisen, E., 1926. The state of a solid body, DTIC Document, Springer, Berlin. volume 10, pp. 1-52. Translated: (1959) The State of a Solid Body (Nat. Aeronautics Space Admin., Washington, DC), NASA Publ. No. RE2-18-59W, pp.1-76.

Guillot, B., Sarda, P., 2006. The effect of compression on noble gas solubility in silicate melts and consequences for degassing at mid-ocean ridges. Geochimica et Cosmochimica Acta 70, 1215-1230. 
Guillot, B., Sator, N., 2007. A computer simulation study of natural silicate melts. Part I: Low pressure properties. Geochimica et Cosmochimica Acta 71, 1249-1265.

Guillot, B., Sator, N., 2012. Noble gases in high-pressure silicate liquids: A computer simulation study. Geochimica et Cosmochimica Acta 80, 51-69.

Guo, X., Lange, R.A., Ai, Y., 2013. The density and compressibility of CaO-FeO-SiO2 liquids: evidence for four-coordinated $\mathrm{Fe} 2+$ in the $\mathrm{CaFeO} 2$ component. Geochimica et Cosmochimica Acta (submitted).

Hemley, R.J., 1987. Pressure Dependence of Raman Spectra of SiO2 Polymorphs: Quartz, Coesite, and Stishovite, in: High-Pressure Research in Mineral Physics: A Volume in Honor of Syun-iti Akimoto. American Geophysical Union, pp. 347-359.

Hoppe, R., 1966. Madelung Constants. Angew. Chem. Int. Ed. Engl. 5, 95-106.

Hoppe, R., 1979. Effective coordination numbers (ECoN) and mean Active fictive ionic radii (MEFIR)[1,2]*. Zeitschrift fr Kristallographie 150, 23-52.

Jaffe, J.E., Snyder, J.A., Lin, Z., Hess, A.C., 2000. LDA and GGA calculations for high-pressure phase transitions in $\mathrm{ZnO}$ and MgO. Phys. Rev. B 62, 1660-1665.

Jing, Z., Karato, S.i., 2011. A new approach to the equation of state of silicate melts: An application of the theory of hard sphere mixtures. Geochimica et Cosmochimica Acta 75, 6780-6802.

Joshi, K., Sharma, B., Paliwal, U., Barbiellini, B., 2012. Pressure-dependent electronic properties of $\mathrm{MgO}$ polymorphs: a first-principles study of Compton profiles and autocorrelation functions. Journal of Materials Science , 1-9.

Karki, B., 1997. Structure and elasticity of $\mathrm{MgO}$ at high pressure. American Mineralogist $82,51-60$.

Karki, B.B., 2015. First-principles computation of mantle materials in crystalline and amorphous phases. Physics of the Earth and Planetary Interiors 240, 43-69. 
Karki, B.B., Bhattarai, D., Stixrude, L., 2006. First-principles calculations of the structural, dynamical, and electronic properties of liquid MgO. Phys. Rev. B 73, 174208-.

Karki, B.B., Bhattarai, D., Stixrude, L., 2007. First-principles simulations of liquid silica: Structural and dynamical behavior at high pressure. Phys. Rev. B 76, 104205-

Kieffer, S.W., 1976. Lattice thermal conductivity within the Earth and considerations of a relationship between the pressure dependence of the thermal diffusivity and the volume dependence of the Grüneisen parameter. J. Geophys. Res. 81, 3025-3030.

Knittle, E., Wentzcovitch, R.M., Jeanloz, R., Cohen, M.L., 1989. Experimental and theoretical equation of state of cubic boron nitride. Nature 337, 349-352.

Knopoff, L., Shapiro, J.N., 1970. Pseudo-Grüneisen Parameter for Liquids. Phys. Rev. B 1, 3893-3895.

de Koker, N., Karki, B.B., Stixrude, L., 2013. Thermodynamics of the MgO-SiO2 liquid system in Earth's lowermost mantle from first principles. Earth and Planetary Science Letters 361, 58-63.

de Koker, N., Stixrude, L., 2009. Self-consistent thermodynamic description of silicate liquids, with application to shock melting of $\mathrm{MgO}$ periclase and $\mathrm{MgSiO} 3$ perovskite. Geophysical Journal International 178, 162-179.

de Koker, N.P., Stixrude, L., Karki, B.B., 2008. Thermodynamics, structure, dynamics, and freezing of $\mathrm{Mg} 2 \mathrm{SiO} 4$ liquid at high pressure. Geochimica et Cosmochimica Acta $72,1427-1441$.

Kruh, R.F., Clayton, G.T., Head, C., Sandlin, G., 1963. Structure of Liquid Mercury. Phys. Rev. 129, 1479-1480.

Lebowitz, J.L., 1964. Exact solution of generalized Percus-Yevick equation for a mixture of hard spheres. Phys. Rev. 133, A895-A899. 
Lee, S.K., 2011. Simplicity in melt densification in multicomponent magmatic reservoirs in Earths interior revealed by multinuclear magnetic resonance. Proceedings of the National Academy of Sciences 108, 6847-6852.

Leinenweber, K., Navrotsky, A., 1988. A transferable interatomic potential for crystalline phases in the system $\mathrm{MgO}-\mathrm{SiO} 2$ 15, 588-596-.

Mansoori, G.A., Canfield, F.B., 1969. Variational Approach to the Equilibrium Thermodynamic Properties of Simple Liquids. I. J. Chem. Phys. 51, 4958-4967.

Mansoori, G.A., Carnahan, N.F., Starling, K.E., Leland, Jr., T.W., 1971. Equilibrium Thermodynamic Properties of the Mixture of Hard Spheres. J. Chem. Phys. 54, $1523-1525$.

Matsui, M., 1994. A transferable interatomic potential model for crystals and melts in the system $\mathrm{CaO}-\mathrm{MgO}-\mathrm{Al} 2 \mathrm{O} 3-\mathrm{SiO} 2$. Mineral Mag 58A, 571-572.

Matsui, M., 1996. Molecular dynamics study of the structures and bulk moduli of crystals in the system CaO-MgO-Al2O3-SiO2 23, 345-353-.

McQueen, R.G., Marsh, S.P., 1960. Equation of State for Nineteen Metallic Elements from ShockWave Measurements to Two Megabars. Journal of Applied Physics 31, 1253-1269.

van Meel, J.A., Filion, L., Valeriani, C., Frenkel, D., 2012. A parameter-free, solidangle based, nearest-neighbor algorithm. J. Chem. Phys. 136, 234107-12.

Momma, K., Izumi, F., 2011. VESTA 3 for three-dimensional visualization of crystal, volumetric and morphology data. Journal of Applied Crystallography 44, 12721276.

Mosenfelder, J.L., Asimow, P.D., Frost, D.J., Rubie, D.C., Ahrens, T.J., 2009. The MgSiO3 system at high pressure: Thermodynamic properties of perovskite, postperovskite, and melt from global inversion of shock and static compression data. J. Geophys. Res. 114, B01203-. 
Mysen, B., Richet, P., 2005. Silicate glasses and melts: properties and structure. volume 10. Elsevier.

Nakajima, M., Stevenson, D.J., 2014. Investigation of the initial state of the Moonforming disk: Bridging SPH simulations and hydrostatic models. Icarus 233, 259267.

Oganov, A.R., Brodholt, J.P., Price, G., 2001. Ab initio elasticity and thermal equation of state of MgSiO3 perovskite. Earth and Planetary Science Letters 184, 555-560.

Ohtani, E., 1983. Melting temperature distribution and fractionation in the lower mantle. Physics of the Earth and Planetary Interiors 33, 12-25.

Ohtani, E., 2009. Melting relations and the equation of state of magmas at high pressure: Application to geodynamics. Chemical Geology 265, 279-288.

Percus, J.K., Yevick, G.J., 1958. Analysis of Classical Statistical Mechanics by Means of Collective Coordinates. Phys. Rev. 110,1-13.

Muñoz Ramo, D., Stixrude, L., 2014. Spin crossover in Fe2SiO4 liquid at high pressure. Geophys. Res. Lett. , n/a-n/a.

Roberts, R.W., Ruppin, R., 1971. Volume Dependence of the Grüneisen Parameter of Alkali Halides. Phys. Rev. B 4, 2041-2046.

Roy, D., Manna, A., Gupta, S.P.S., 1974. The application of the Morse potential function in ordered $\mathrm{Cu} 3 \mathrm{Au}$ alloy. II. Thermal expansion and the equation of state. Journal of Physics F: Metal Physics 4, 2145-.

Sanloup, C., Drewitt, J.W.E., Konopkova, Z., Dalladay-Simpson, P., Morton, D.M., Rai, N., van Westrenen, W., Morgenroth, W., 2013. Structural change in molten basalt at deep mantle conditions. Nature 503, 104-107. W14.

Schleife, A., Fuchs, F., Furthmller, J., Bechstedt, F., 2006. First-principles study of ground- and excited-state properties of $\mathrm{MgO}, \mathrm{ZnO}$, and $\mathrm{CdO}$ polymorphs. Phys. Rev. B 73, 245212-. 
Shen, G., Prakapenka, V.B., Rivers, M.L., Sutton, S.R., 2004. Structure of Liquid Iron at Pressures up to 58GPa. Phys. Rev. Lett. 92, 185701-.

Soga, N., Anderson, O.L., 1966. High-Temperature Elastic Properties of Polycrystalline $\mathrm{MgO}$ and Al2O3. Journal of the American Ceramic Society 49, 355-359.

Spera, F.J., Ghiorso, M.S., Nevins, D., 2011. Structure, thermodynamic and transport properties of liquid $\mathrm{MgSiO} 3$ : Comparison of molecular models and laboratory results. Geochimica et Cosmochimica Acta 75, 1272-1296.

Stebbins, J.F., Murdoch, J.B., Schneider, E., Carmichael, I.S.E., Pines, A., 1985. A high-temperature high-resolution NMR study of 23Na, 27Al and 29Si in molten silicates. Nature 314, 250-252.

Stixrude, L., Karki, B., 2005. Structure and Freezing of MgSiO3 Liquid in Earth's Lower Mantle. Science 310, 297-299.

Stixrude, L., de Koker, N., Sun, N., Mookherjee, M., Karki, B.B., 2009. Thermodynamics of silicate liquids in the deep Earth. Earth and Planetary Science Letters 278, 226-232.

Thomas, C.W., Liu, Q., Agee, C.B., Asimow, P.D., Lange, R.A., 2012. Multi-technique equation of state for $\mathrm{Fe} 2 \mathrm{SiO} 4 m e l t$ and the density of Fe-bearing silicate melts from 0 to 161 GPa. J. Geophys. Res. 117, B10206-.

Trachenko, K., Brazhkin, V.V., 2013. Duality of liquids. Sci. Rep. 3, -.

Vocadlo, L., Alf, D., Gillan, M., Price, G., 2003. The properties of iron under core conditions from first principles calculations. Physics of the Earth and Planetary Interiors 140, 101-125.

Van de Walle, A., Ceder, G., 1999. Correcting overbinding in local-densityapproximation calculations. Physical Review B 59, 14992.

Wang, Y., Sakamaki, T., Skinner, L.B., Jing, Z., Yu, T., Kono, Y., Park, C., Shen, G., Rivers, M.L., Sutton, S.R., 2014. Atomistic insight into viscosity and density of silicate melts under pressure. Nat Commun 5, -. W14. 
Welche, P.R.L., Heine, V., Dove, M.T., 1998. Negative thermal expansion in betaquartz. Physics and Chemistry of Minerals 26, 63-77-.

Williams, Q., Hemley, R.J., Kruger, M.B., Jeanloz, R., 1993. High-pressure infrared sepctra of -quartz, coesite, stishovite and silica glass. J. Geophys. Res. 98, 2215722170.

Xue, X., Stebbins, J.F., Kanzaki, M., McMillan, P.F., Poe, B., 1991. Pressure-induced silicon coordination and tetrahedral structural changes in alkali oxide-silica melts up to $12 \mathrm{GPa}$; NMR, Raman, and infrared spectroscopy. American Mineralogist 76, $8-26$.

Yamada, A., Inoue, T., Urakawa, S., Funakoshi, K.i., Funamori, N., Kikegawa, T., Irifune, T., 2011. In situ X-ray diffraction study on pressure-induced structural changes in hydrous forsterite and enstatite melts. Earth and Planetary Science Letters 308, $115-123$.

Yarger, J.L., Smith, K.H., Nieman, R.A., Diefenbacher, J., Wolf, G.H., Poe, B.T., McMillan, P.F., 1995. Al Coordination Changes in High-Pressure Aluminosilicate Liquids. Science 270, 1964-1967.

Yuste, S.B., Santos, A., de Haro, M.L., 1998. Structure of multi-component hardsphere mixtures. J. Chem. Phys. 108, 3683-3693.

\section{Appendix A. Generalized Hard Sphere Mixture}

The complete set of expressions for the equation of state of a hard sphere mixture is presented in Mansoori et al. (1971). For completeness, we include these expressions here, which represent the total free energy for a hard sphere mixture as a sum of terms:

$$
F^{\mathrm{HS}}=F^{\mathrm{gas}}+F^{\mathrm{mix}}+\Delta F^{\mathrm{HS}}
$$

where $F^{\text {gas }}$ is the standard ideal gas contribution (see Equation A.2), $F^{\text {mix }}$ is the entropic mixing contribution associated with random mixing of liquid particles (see Equation 
A.3), and $\Delta F^{\mathrm{HS}}$ is the adjustment to the ideal gas accounting for the finite volume occupied by liquid particles or ionic clusters (see Equation A.7).

The two ideal contributions are the ideal gas and ideal mixing terms. The free energy of an ideal gas is:

$$
\begin{aligned}
F^{\mathrm{gas}} & =-k_{B} T \log _{e} Q^{\mathrm{gas}} \\
\log _{e} Q^{\mathrm{gas}} & =\log _{e} V+\frac{3}{2} \log _{e}\left(\frac{m k_{B} T}{2 \pi}\right)+1
\end{aligned}
$$

where $k_{B}$ is Boltzmann's constant, $Q^{\text {gas }}$ is the canonical partition function of the ideal gas, and the mass $m$ and volume $V$ are expressed in atomic units (e.g., proton masses and $\left.b o h r^{3}\right)$. The entropic mixing contribution to the free energy is:

$$
\begin{aligned}
& F^{\text {mix }}=-T S^{\text {mix }} \\
& S^{\text {mix }}=-k_{B} \sum_{j}^{\mathrm{CN}} \bar{p}_{j} \log _{e} \bar{p}_{j}
\end{aligned}
$$

This is just the ideal entropy of mixing for the set of integer coordination states, where $\bar{p}_{j}$ refers to the binned population of the $j^{\text {th }}$ coordination state.

The analytic form of the hard sphere free energy correction, which accounts for the volume occupied by the hard spheres, is somewhat complex but easily evaluated. We begin with the expression for the packing fraction:

$$
\eta_{i} \equiv \frac{V_{i}}{V}=p_{i} \frac{\pi}{6 V} d_{i}^{3} \quad \text { where } \quad \eta=\sum_{i}^{\mathrm{HS}} \eta_{i}
$$

where $p_{i}$ and $d_{i}$ are the population and diameter of the $i^{\text {th }}$ hard sphere, and $\eta_{i}$ is its contribution to the total packing fraction. We must also define the compressibility factor $Z$, expressed as a function of the packing fraction:

$$
Z=\frac{\hat{P} V}{k_{B} T} \approx\left[1+\eta+\eta^{2}-3 \eta\left(y_{1}+y_{2} \eta\right)-\eta^{3} y_{3}\right](1-\eta)^{-3}
$$


where the needed additional relations are:

$$
\begin{array}{r}
y_{1}=\sum_{i=1} \sum_{j>i} \Delta_{i j}\left(d_{i}+d_{j}\right)\left(d_{i} d_{j}\right)^{-1 / 2} \\
y_{2}=\sum_{i=1} \sum_{j>i} \Delta_{i j} \sum_{k=1}^{N}\left(\frac{\eta_{k}}{\eta}\right) \frac{\left(d_{i} d_{j}\right)^{1 / 2}}{d_{k}} \\
y_{3}=\left[\sum_{i=1}\left(\frac{\eta_{i}}{\eta}\right)^{2 / 3} p_{i}^{1 / 3}\right]^{3} \\
\Delta_{i j}=\left[\frac{\left(\eta_{i} \eta_{j}\right)^{\frac{1}{2}}}{\eta}\right]\left[\frac{\left(d_{i}-d_{j}\right)^{2}}{d_{i} d_{j}}\right]\left(p_{i} p_{j}\right)^{\frac{1}{2}}
\end{array}
$$

It is important to note that while symbol $\hat{P}$ has the units of pressure, it refers to the true pressure only if we are considering the basic hard sphere model where the hard sphere populations and sizes are fixed. Otherwise, hard sphere evolution introduces additional pressure terms not accounted for in this expression.

These are all combined to express the contribution to the free energy of the liquid:

$$
\begin{gathered}
\frac{\Delta F_{H S}}{k_{B} T}=-\frac{3}{2}\left(1-y_{1}+y_{2}+y_{3}\right)+\left(3 y_{2}+2 y_{3}\right)(1-\eta)^{-1}+ \\
\frac{3}{2}\left(1-y_{1}-y_{2}-\frac{1}{3} y_{3}\right)(1-\eta)^{-2}+\left(y_{3}-1\right) \log _{e}(1-\eta)
\end{gathered}
$$

By combining all of the expressions in this section through Equation (A.1), we can evaluate the total contributions to the free energy from the standard hard sphere mixture model. If these expressions are used within a variational scheme where evolving hard sphere sizes and populations are determined by free energy minimization (e.g., Equation 8 ), then pressure and entropy must be determined numerically from the thermodynamic derivatives $P=-\partial F / \partial V_{T}$ and $S=-\partial F / \partial T_{V}$.

\section{Appendix B. Hard Spheres with Structural Perturbation}

The variational approach to hard spheres allows the incorporation of a structural perturbation into the free energy expression, which captures the dependence of the internal energy on the structure of the liquid. This variational method was developed by Mansoori and Canfield (1969), and the relevant expressions are reproduced below.

In the standard variational Hard Sphere approach (Equation 3), the structural energy contribution is approximated using the radial distribution function $g(r)$, together with 
the pair potential $u(r)$ :

$$
E^{\mathrm{struc}}=\frac{1}{2} \int_{0}^{\infty} \mathcal{P}(r) u(r) d r=2 \pi \rho \int_{d_{\mathrm{HS}}}^{\infty} r u(r) \cdot r g(r) d r
$$

Mansoori and Canfield (1969) showed that the above integral for the structural perturbation energy $E^{\text {struc }}$ can be simplified using a Laplace transformation:

$$
E^{\text {struc }}=2 \pi \rho \int_{0}^{\infty} U(s) G(s) d s
$$

where $G(s)=\mathcal{L}\{r g(r)\}$ and $U(s)=\mathcal{L}^{-1}\{r u(r)\}$ are the Laplace and inverse Laplace transforms of the expressions involving the RDF and pair potential functions, respectively. The expression for $G(s)$ has a simple functional form given by Percus-Yevick theory (e.g. Percus and Yevick, 1958; Lebowitz, 1964; Yuste et al., 1998):

$$
\begin{aligned}
G(s) & =\frac{\exp \left(-s d_{\mathrm{HS}}\right)}{2 \pi s^{2}} \frac{L^{(0)}+s L^{(1)}}{1-1 / V \cdot\left[\phi_{2}\left(s d_{\mathrm{HS}}\right) \cdot d_{\mathrm{HS}}^{3} L^{(0)}+\phi_{1}\left(s d_{\mathrm{HS}}\right) \cdot d_{\mathrm{HS}}^{2} L^{(1)}\right]} \\
\phi_{n}(x) & \equiv x^{-(n+1)}\left(\sum_{m=0}^{n} \frac{(-x)^{m}}{m !}-\exp (-x)\right) \\
L^{(0)} & =2 \pi \frac{1+2 \eta}{(1-\eta)^{2}} \\
L^{(1)} & =2 \pi d_{\mathrm{HS}} \frac{1+\frac{1}{2} \eta}{(1-\eta)^{2}}
\end{aligned}
$$

Using this expression, we can take a numerical inverse Laplace transform of $G(s)$ to calculate the radial distribution function. Even more efficient, however, is to take advantage of the form of the Morse potential in order to yield extremely rapid structural energy calculations.

The Morse potential (Equation XXX) can be rewritten in the simple form

$$
E_{\text {Morse }}=A_{r} \exp \left(-k_{r} r\right)-A_{a} \exp \left(-k_{a} r\right)
$$

where $r$ and $a$ subscripts refer to the contributions of the repulsive and attractive interactions, respectively. The proper values of these parameters are determined as a function of coordination number based on the cluster potential model reported in Table 1. Using standard transform tables, we determine the inverse Laplace transform of $r E_{\text {Morse }}(r)$ :

$$
U(s)=A_{r} \delta^{\prime}\left(s-k_{r}\right)-A_{a} \delta^{\prime}\left(s-k_{a}\right)
$$


where $\delta^{\prime}(x-b)$ is the derivative delta function, which returns the derivative of the function it operates on evaluated at $x=b$. Thus we can now trivially compute the structural energy integral:

$$
E^{\mathrm{struc}}=2 \pi \rho \int_{0}^{\infty}\left[A_{r} \delta^{\prime}\left(s-k_{r}\right)-A_{a} \delta^{\prime}\left(s-k_{a}\right)\right] G(s) d s=2 \pi \rho\left[-A_{r} G^{\prime}\left(k_{r}\right)+A_{a} G^{\prime}\left(k_{a}\right)\right] \text { (B.6) }
$$

This expression shows that the total structural energy can be determined for a Morse potential by evaluating $G(s)$, Equation B.3, at a few discrete points to calculate the numerical derivative, in place of a costly inverse Laplace transform followed by numerical integration.

\section{Appendix C. Bond Energy Model}

In an oxide or silicate melt, variations in bond distance and bond angle occur due to the vibrational motions of individual ions, which contribute to the overall free energy of the melt. We incorporate these entropic sources of free energy into the CHaSM model as the internal oscillator correction term, $F^{\text {osc }}$ found in Equation (6), which accounts for the internal degrees of freedom available to the individual ions within each ionic cluster. In order to derive the value of $F^{\text {osc }}$, we must first obtain a reasonable description of the vibrational energy resulting from the individual ions. We therefore construct a bond energy model that accounts for variations in internal energy due to $\mathrm{Mg}-\mathrm{O}$ bond length changes.

In this section, we derive a structural energy description of $\mathrm{MgO}$ in terms of its bonding characteristics, by fitting the energy volume curves of the $\mathrm{MgO}$ polymorphs with a coordination-dependent bond potential. We use the basic Born ionic potential form:

$$
E_{\mathrm{bond}}=E_{R}-E_{\mathrm{coul}}=\mathrm{CN} \cdot A_{r}^{\text {bond }}\left(\frac{d_{\mathrm{bond}}}{a_{0}}\right)^{-n_{r}}-E_{\mathrm{coul}}+\Delta E_{0}
$$
where $\mathrm{CN}$ is the cation coordination number, $n_{r}$ is repulsive exponent, $A_{r}^{\text {bond }}$ scales the strength of the repulsive interaction, and $d_{\text {bond }}$ is the $\mathrm{Mg-O}$ bond length, scaled by the Bohr radius $a_{0}=0.529 \AA$, and $\Delta E_{0}$ is an arbitrary energy offset parameter that is shared by all polymorphs. For crystalline solids, the Coulomb energy contribution is readily 
Table C.2: Best-fit Coordination-Dependent Bond-Energy Potential for $\mathrm{MgO}$

\begin{tabular}{lccc}
\hline $\boldsymbol{\theta}_{\text {bond }}$ & $\boldsymbol{k}_{\mathbf{2}}$ & $\boldsymbol{k}_{\mathbf{1}}$ & $\boldsymbol{k}_{\mathbf{0}}$ \\
\hline $\log _{e} A_{r}^{\text {bond }}$ & +0.0398 & +0.1345 & +6.8563 \\
$n_{r}$ & +0.0219 & +0.1456 & +4.5055 \\
\hline
\end{tabular}

971

evaluated using the Madelung lattice energy

$$
E_{\text {coul }}=\frac{q^{2} M}{d_{\text {bond }} / a_{0}}
$$

where $q=2$ is the ionic charge, and $M$ is the Madelung constant, which takes typical values from 1 to 1.8 , depending on coordination and the detailed structure of the solid (Hoppe, 1966).

We fit the bond-potential model to the $\mathrm{MgO}$ polymorph data, assuming that the parameter values $\boldsymbol{\theta}_{\mathrm{bond}}=\left(\log _{e} A_{r}^{\text {bond }}, n_{r}\right)$ depend quadratically on coordination number

$$
\theta_{\text {bond }}=\boldsymbol{k}_{2} \Delta \mathrm{CN}^{2}+\boldsymbol{k}_{1} \Delta \mathrm{CN}+\boldsymbol{k}_{\mathbf{0}}
$$

where $\Delta \mathrm{CN}=C N-6$. The quality of the resulting fit is similar to that of the cluster potential model, with best-fit values given in Table C. $2^{4}$.

\section{Appendix D. Vibrational Motions}

To directly connect the hard sphere model to the atomic structure of oxide melts, we account not only for the configurational entropy contribution to the free energy, but also the added vibrational component due to the individual ions. This free energy correction is represented by the internal oscillation term, $F^{\text {osc }}$, in the CHaSM free energy expression (Equation 6). To derive the expression for this term, we must first obtain the vibrational frequencies of the cluster and bond-energy potential models.

\footnotetext{
${ }^{4}$ Though the bond potential form is similar to the classic Born-Landé approximation for lattice energy, the parameter values differ due to the introduction of $\Delta E_{0}$, which allows us to better represent compressibilities and vibrational properties, rather than absolute lattice energies.
} 
We rely on the approximate expression for restoring force-constants given in Equation (15), derived by taking a second derivative of the structural energy with respect to displacements of the particle from equilibrium, where the sum is replaced by an integral to better reflect liquid structure:

$$
\begin{aligned}
\hat{k}_{\mathrm{MgO}[\mathrm{CN}]}=m_{\mathrm{MgO}} \cdot \omega_{\mathrm{MgO}}^{2}[\mathrm{CN}] & \approx \frac{4 \pi}{V} \int_{d_{\mathrm{HS}}}^{\infty} r \hat{k}_{[\mathrm{CN}]}(r) \cdot r g_{[\mathrm{CN}]}(r) d r \\
\hat{k}_{[\mathrm{CN}]}(r) & =\frac{1}{6}\left[E_{\mathrm{pot}}^{\prime \prime}(r, \mathrm{CN})+\frac{2}{r} E_{\mathrm{pot}}^{\prime}(r, \mathrm{CN})\right]
\end{aligned}
$$

where $\hat{k}_{\mathrm{MgO}} \mathrm{O}^{[\mathrm{CN}]}$ is the total effective restoring force constant acting on ionic cluster particles with cation coordination of $\mathrm{CN}, \hat{k}_{[\mathrm{CN}]}(r)$ is the contribution to the restoring force due to interactions at a distance $r$, and $E_{\mathrm{pot}}(r, C N)$ defines the coordinationdependent cluster potential energy interaction. This expression estimates a representative restoring force constant and frequency for individual ionic clusters, akin to the Einstein model, and is thus incapable of representing true phonon modes involving many particles. In this calculation, the local environment of each particle, given by the $\mathrm{RDF} g_{[\mathrm{CN}]}(r)$, is held fixed and is thus assumed uncorrelated with the particle vibration. Despite these approximations, this expression is nevertheless useful for obtaining a rough estimate of the oscillatory motions within the liquid, and is in fact considerably more accurate for liquids than it is for solids, since the long-wavelength phonon modes that are neglected do not play an important role in liquid energetics due to the lack of long-range order.

We can simplify this expression for the Morse cluster potential using the same Laplace transform approach used in Section Appendix B, taking the inverse Laplace transform of the $r \hat{k}(r)$ term, yielding:

$$
\begin{aligned}
& \hat{k}_{\mathrm{MgO} \mathrm{O}^{[\mathrm{CN}]}}=\frac{2 \pi}{3} \rho d_{\mathrm{HS}}^{3}\left\{\int_{0}^{\infty}\left[-k_{r}^{2} A_{r} \delta^{\prime}\left(s-k_{r}\right)+k_{a}^{2} A_{a} \delta^{\prime}\left(s-k_{a}\right)\right] \cdot G(s) d s\right. \\
& \left.-\frac{1}{d_{\mathrm{HS}}} \int_{0}^{\infty}\left[+k_{r} A_{r} \delta\left(s-k_{r}\right)-k_{a} A_{a} \delta\left(s-k_{a}\right)\right] \cdot G(s) d s\right\}
\end{aligned}
$$

$$
\hat{k}_{\mathrm{MgO}}{ }^{[\mathrm{CN}]}=\frac{2 \pi}{3} \rho d_{\mathrm{HS}}^{3}\left\{-k_{r}^{2} A_{r} G^{\prime}\left(k_{r}\right)+k_{a}^{2} A_{a} G^{\prime}\left(k_{a}\right)-\frac{k_{r}}{d_{\mathrm{HS}}} A_{r} G\left(k_{r}\right)+\frac{k_{a}}{d_{\mathrm{HS}}} A_{a} G\left(k_{a}\right)\right\}
$$

where the simplified Morse potential parameters $\left(A_{r}, k_{r}, A_{a}, k_{a}\right)$ are defined by Equation B.4, and $G(s)$ is the Laplace transform of the hard sphere RDF, given by Equation 
(B.3). This expression is rapidly evaluated just as the structural energy given by Equation (B.6).

Finally, we must also determine the restoring force constant for the bond energy model, which describes the vibrations of individual ions in response to bond stretching. When considering displacements of individual ions, we use the bond energy equation (C.1), and consider the total Coulombic attraction of each of the coordinated ions $\left|E_{\text {coul }}\right|=\mathrm{CN} \cdot q^{2} /\left(r / a_{0}\right)$, forgoing the use of the Madelung constant since we neglect such longer-range interactions for the disordered liquid (and they contribute much less to the curvature anyway). Since the bond energy model relies only on directly bonded ions, we can use a simplified discrete form of the restoring force equation:

$$
\hat{k}_{\mathrm{bond}}=\frac{1}{6}\left[E_{\mathrm{bond}}^{\prime \prime}(r)+\frac{2}{r} E_{\mathrm{bond}}^{\prime}(r)\right]_{r=d_{\mathrm{bond}}}
$$

Since the form of $E_{\text {bond }}$ is just a simple polynomial in $1 / r$, evaluation of this expression is trivial using the coordination dependent bond energy parameters given in Table C.2. Though this expression neglects longer-range interactions beyond bonded neighbors, this approximation is not unreasonable for liquids, and thus should provide a decent first-order estimate of the vibrational properties of the individual ions.

\section{Appendix E. Internal Oscillation Correction}

Up to this point, we have presented two alternate models for the oxide melt system, one in terms of ionic clusters and the other using bond energies (Appendix BAppendix D). The clear advantage of the ionic cluster model is that it enables the use of hard sphere theory to represent liquid structure and configurational entropy, but has the downside of not accounting for the independent motions of the ions within each cluster. To overcome this issue, we introduce an additional internal oscillator term, $F^{\text {osc }}$, which represents the missing part of the vibrational free energy. To derive the expression for $F^{\text {osc }}$, we begin with the quasi-harmonic expression for vibrational free energy:

$$
F^{\mathrm{qha}}=\sum_{i}\left\{\frac{1}{2} \hbar \omega_{i}+k_{B} T \log _{e}\left[1-\exp \left(-\frac{\hbar \omega_{i}}{k_{B} T}\right)\right]\right\}
$$


where $\omega_{i}$ is the frequency of the $i^{\text {th }}$ vibrational mode. This expression simplifies in the high temperature limit to:

$$
F_{i}^{\mathrm{vib}} \approx 3 k_{B} T \log _{e}\left(\frac{\hbar \omega_{i}}{k_{B} T}\right)
$$

where the factor of 3 reflects the three independent oscillation directions. The vibrational frequency is simply determined from the restoring force constant $\hat{k}$ and the particle mass $m$, given by $\omega=\sqrt{\hat{k} / m}$.

We can now compare the total approximate vibrational free energy (using Equation E.2) for the two alternative vibrational models of the system:

$$
\begin{aligned}
\frac{F_{\mathrm{Tot}}^{\mathrm{vib}}}{3 k_{B} T} & \approx \frac{1}{2} \log _{e}\left(\frac{\hat{k}_{\mathrm{bond}}}{m_{\mathrm{Mg}}}\right)+\frac{1}{2} \log _{e}\left(\frac{\hat{k}_{\mathrm{bond}}}{m_{\mathrm{O}}}\right)+\log _{e}\left(\frac{\hbar}{k_{B} T}\right) \quad \text { Bond Energy } \\
& \approx \frac{1}{2} \log _{e}\left(\frac{\hat{k}_{\mathrm{MgO}}^{[\mathrm{CN}]}}{m_{\mathrm{MgO}}}\right)+\frac{1}{2} \log _{e}\left(\frac{\hat{k}_{\mathrm{osc}}}{m_{\mathrm{osc}}}\right)+\log _{e}\left(\frac{\hbar}{k_{B} T}\right) \quad \text { Ionic Cluster }
\end{aligned}
$$

where the first line expresses the individual atomic vibrations using the bond-energy model, and the second line provides the CHaSM-derived free energy in terms of the cluster vibrations and the effective internal oscillations. The restoring force constants $\hat{k}_{\text {bond }}$ and $\hat{k}_{\mathrm{MgO}^{[\mathrm{CN}]}}$ are given by Equations (D.3) and (D.2), and thus we can solve for internal oscillator character characteristics:

$$
\begin{gathered}
\hat{k}_{\mathrm{osc}}=\hat{k}_{\mathrm{bond}}^{2} / \hat{k}_{\mathrm{MgO}^{[\mathrm{CN}]}}, \quad m_{\mathrm{osc}}=m_{\mathrm{Mg}} m_{\mathrm{O}} / m_{\mathrm{MgO}}, \\
\omega_{\mathrm{osc}}=\sqrt{\hat{k}_{\mathrm{osc}} / m_{\mathrm{osc}}}
\end{gathered}
$$

where the internal oscillator mass and force constant both have a similar form, and the mass is simply the reduced mass of the ionic pair. Finally, we can evaluate the quasi-harmonic model for a three-dimensional oscillator with frequency $\omega_{\mathrm{osc}}$ :

$$
F^{\mathrm{osc}}=\frac{3}{2} \hbar \omega_{\mathrm{osc}}+3 k_{B} T \log _{e}\left[1-\exp \left(-\frac{\hbar \omega_{\mathrm{osc}}}{k_{B} T}\right)\right]
$$

By combining this expression for $F^{\text {osc }}$ with those for the structural energy $E^{\text {struc }}$ (Equation B.6) and the hard sphere free energy $F^{\mathrm{HS}}$ (Equation A.1), we have all of the pieces needed to evaluate the CHaSM free energy model. 


\section{Appendix F. Fractional Coordination Numbers and Bond Lengths}

For non-ideal or distorted structures, the consideration of fractional coordination numbers can be useful in examining geometric trends with coordination environment. Furthermore, the calculation of average ion-pair distances like bond lengths requires a clear understanding of how to weight neighboring ions that occupy a range of distances. This topic was examined in detail by Hoppe (1979), who used an exponential averaging scheme to give greater weight to closer neighbors in determining effective coordination numbers and average bond lengths. In this work, we make use of a more recent alternative weighting scheme, the Solid Angle Nearest Neighbor (SANN) method (van Meel et al., 2012), which provides geometrically intuitive estimates that better reflect the differences between the structures examined in this study.

The SANN method identifies the relative importance of neighboring atoms as proportional to the apparent solid angle, $\Omega_{i}$, they present to the central atom:

$$
\Omega_{i}=2 \pi\left[1-\cos \theta_{i}\right]=2 \pi\left(1-r_{i} / R\right)
$$

where the set of neighboring atoms are considered to lie within a neighborhood shell of radius $R$, and $r_{i}$ is the distance to the $i^{\text {th }}$ neighbor. The size of the neighborhood is determined by requiring that the total solid angle of all $m$ neighbors is complete, with $4 \pi=\sum_{i}^{m} \Omega_{i}$. The neighborhood shell radius can thus be determined with:

$$
\left(R=\sum_{i}^{m} \frac{r_{i}}{(m-2)}\right)<r_{m+1}
$$

where the number of neighbors $m$ is increased until the inequality criterion is met.

Extending this procedure from van Meel et al. (2012), we define the coordination weight of each neighbor as the solid angle relative to that of the closest neighbor:

$$
\mathrm{CN}_{\mathrm{SA}}=\sum_{i}^{m} w_{i} \quad \text { with } \quad w_{i}=\frac{\Omega_{i}}{\Omega_{1}}=\frac{1-r_{i} / R}{1-r_{1} / R}
$$

where the total coordination number is just the sum of the weights. This definition is trivial to calculate, parameter free, and yields the intuitive result that the coordination weight for the nearest neighbor is defined to be one. Using this coordination definition based on solid angles, we recover the expected values for ideal structures as well 
1074 as finding smooth systematic behavior for the set of progressively more coordinated 1075 structures of $\mathrm{MgO}$. We can also use this coordination weighting factor to determine other quantities of interest through weighted averaging, like the average bond length

$1077\left\langle d_{\mathrm{bond}}\right\rangle=\sum_{i} w_{i} r_{\mathrm{Mg}-\mathrm{O}, i}$ or cation separation distance $\left\langle d_{\mathrm{Mg}-\mathrm{Mg}}\right\rangle=\sum_{i} w_{i} d_{\mathrm{Mg}-\mathrm{Mg}, i}$. This 1078 minor extension of the Solid Angle Nearest Neighbor method provides a simple and 1079 powerful tool for characterizing local atomic structure. 